\title{
The embedding theorem for finite depth subfactor planar algebras
}

\author{
Vaughan F. R. Jones and David Penneys ${ }^{1}$
}

\begin{abstract}
We define a canonical planar *-algebra from a strongly Markov inclusion of finite von Neumann algebras. In the case of a connected unital inclusion of finite dimensional $C^{*}$-algebras with the Markov trace, we show this planar algebra is isomorphic to the bipartite graph planar algebra of the Bratteli diagram of the inclusion. Finally, we show that a finite depth subfactor planar algebra is a planar subalgebra of the bipartite graph planar algebra of its principal graph.
\end{abstract}

Mathematics Subject Classification (2010). 46L37 (Primary); 18D10, 57M20 (Secondary)

Keywords. Subfactors, planar algebras.

\section{Contents}

1 Introduction . . . . . . . . . . . . . . . 302

2 The canonical planar *-algebra of a strongly Markov inclusion of finite von Neumann algebras . . . . . . . . . . . . . . . 303

2.1 Bases, traces, and strongly Markov inclusions . . . . . . . . . 304

2.2 The Jones tower and tensor products . . . . . . . . . . . . . 307

2.3 Definition of the canonical planar $*$-algebra $\ldots \ldots \ldots . \ldots 312$

2.4 Burns' treatment of the rotation operator on $P_{n,+} \ldots \ldots \ldots 315$

2.5 The rotation on $P_{n,-} \ldots \ldots \ldots \ldots \ldots \ldots$

2.6 Uniqueness of the canonical planar $*$-algebra . . . . . . . . 317

3 The planar algebra isomorphism for finite dimensional $\mathrm{C}^{*}$-algebras . . . . 322

3.1 Loop algebras . . . . . . . . . . . . . . . . . . 322

3.2 Towers of loop algebras . . . . . . . . . . . . . . . . . 325

3.3 Relative commutants are isomorphic to loop algebras . . . . . . . . 327

3.4 The bipartite graph planar algebra and the isomorphism . . . . . . 331

4 The embedding theorem . . . . . . . . . . . . . . . . . 334

References . . . . . . . . . . . . . . . . . . . . . . 336

\footnotetext{
${ }^{1}$ Both authors would like to acknowledge support from NSF grants DMS 0401734 and DMS 0856316 and DOD-DARPA grant HR0011-11-1-0001.
} 


\section{Introduction}

A powerful method of construction of subfactors is the use of commuting squares, which are systems of four finite dimensional von Neumann algebras

$$
\begin{gathered}
A_{1,0} \subset A_{1,1} \\
\cup \quad \cup \\
A_{0,0} \subset A_{0,1}
\end{gathered}
$$

included as above, with a faithful trace on $A_{1,1}$ so that $A_{1,0}$ and $A_{0,1}$ are orthogonal modulo their intersection $A_{0,0}$.

One iterates the basic construction of [8] for the inclusions $A_{i, j} \subset A_{i, j+1}$ and $A_{i, j} \subset A_{i+1, j}$ to obtain a tower of inclusions $A_{0, n} \subset A_{1, n}$. By a lovely compactness argument of Ocneanu, [12] and [5], the standard invariant, or higher relative commutants, of the inductive limit inclusion $A_{0, \infty} \subset A_{1, \infty}$ are the algebras $A_{0,1}^{\prime} \cap A_{n, 0}$. Thus once bases have been chosen, the calculation of the relative commutants is a matter of elementary linear algebra.

It was to formalise this calculation that planar algebras were first introduced [9]. Finite dimensional inclusions are given by certain graphs (Bratteli diagrams), and, in [10], a planar algebra associated purely combinatorially to a bipartite graph was introduced so that it is rather obviously the tower of relative commutants for an inclusion $B_{0} \subset B_{1}$ having the graph as its Bratteli diagram. But because Ocneanu's notion of connection was never completely formalised in [9], it was not proved that the planar algebra coming from a commuting square via Ocneanu compactness is a planar subalgebra of the one defined in [10] for the graph of the inclusion $A_{0,0} \subset A_{1,0}$.

Meanwhile the theory of planar algebras grew in its own right and a new method of constructing subfactors evolved by looking at planar subalgebras of a given planar algebra, [17] and [2]. Now if a subfactor is of finite depth, then by [20], there is a commuting square that constructs a hyperfinite model of it. Moreover the inclusion $A_{0,0} \subset A_{1,0}$ for this canonical commuting square has Bratteli diagram given by the so-called principal graph, which is a powerful subfactor invariant. Thus if the result of the previous paragraph had been proved, it would have implied the following theorem, which is the main result of this paper.

Theorem. A finite depth subfactor planar algebra is a planar subalgebra of the bipartite graph planar algebra of its principal graph.

(See [14] for the definition of the principal graph of a planar algebra.)

We prove this result with the interesting twist of not using connections. In particular, our proof does not invoke the dual principal graph, which is perhaps rather surprising.

There are three steps to our proof. The first step, Section 2, is to define a canonical planar $*$-algebra structure on the tower of relative commutants from a connected unital 
inclusion of finite dimensional $C^{*}$-algebras whose Bratteli diagram is a given graph. We call this the canonical planar $*$-algebra associated to the inclusion. We do this in more generality, replacing finite dimensionality by a strong Markov property (see Definition 2.8), because it is no harder and should have applications.

The second step, Section 3, is to identify the canonical planar $*$-algebra with the bipartite graph planar algebra of [10] in the finite dimensional case. Loops on the Bratteli diagram for the inclusion give bases for the relative commutants, so the isomorphism is constructed by choosing bases for the vector spaces in the canonical planar *-algebra.

Finally, in Section 4, we construct the embedding map as follows: given a finite depth subfactor planar algebra $Q \bullet$, pick $2 r$ suitably large so that the inclusion $Q_{2 r,+} \subset$ $Q_{2 r+1,+} \subset\left(Q_{2 r+2,+}, e_{2 r+1}\right)$ is standard, i.e. isomorphic to the basic construction. Set $M_{0}=Q_{2 r,+}$ and $M_{1}=Q_{2 r+1,+}$, and let $P_{\bullet}$ be the canonical planar $*$-algebra $P_{\bullet}$ associated to the inclusion $M_{0} \subset M_{1}$. We prove in Theorem 4.1 that the map $Q \bullet \rightarrow P$ • given by adding $2 r$ or $2 r+1$ strings on the left, depending on whether we are in $Q_{n,+}$ or $Q_{n,-}$ respectively, is an inclusion of planar algebras.

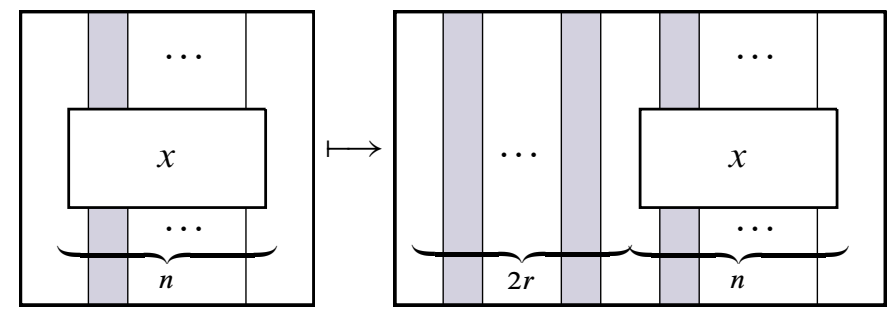

While this paper was being written, Morrison and Walker in [15] produced a totally different proof which constructs an embedding directly from the planar algebra $Q$. without the use of algebra towers and centralisers. Their method also has the advantage that it applies to infinite depth subfactor planar algebras without alteration!

\section{The canonical planar *-algebra of a strongly Markov inclusion of finite von Neumann algebras}

After defining the notion of a strongly Markov inclusion of finite von Neumann algebras, we show the basic construction is also strongly Markov with the same (Watatani) index. We then define the canonical planar $*$-algebra associated to a strongly Markov inclusion.

Many results of this section can be found in [8], [18], [23], [7], [21], [3], and [4], but our treatment differs slightly, so we provide some proofs for the reader's convenience. 


\subsection{Bases, traces, and strongly Markov inclusions}

Notation 2.1. Throughout this paper, a trace on a finite von Neumann algebra means a faithful, normal, tracial state unless otherwise specified. We will write $M_{0} \subset$ $\left(M_{1}, \operatorname{tr}_{1}\right)$ to mean $M_{0} \subset M_{1}$ is an inclusion of finite von Neumann algebras where $\operatorname{tr}_{1}$ is a trace on $M_{1}$. We set $\operatorname{tr}_{0}=\operatorname{tr}_{1} \mid M_{0}$.

Let $M_{0} \subset\left(M_{1}, \operatorname{tr}_{1}\right)$. Let $M_{2}=\left\langle M_{1}, e_{1}\right\rangle=J M_{0}^{\prime} J \subset B\left(L^{2}\left(M_{1}, \operatorname{tr}_{1}\right)\right)$ be the basic construction, where $e_{1}$ is the Jones projection with range $L^{2}\left(M_{0}, \operatorname{tr}_{0}\right)$, and $J: L^{2}\left(M_{1}, \operatorname{tr}_{1}\right) \rightarrow L^{2}\left(M_{1}, \operatorname{tr}_{1}\right)$ is the antilinear unitary given by the antilinear extension of $x \Omega \mapsto x^{*} \Omega$, where $\Omega \in L^{2}\left(M_{1}, \operatorname{tr}_{1}\right)$ is the image of $1 \in M_{1}$.

Recall from [22] that there is a unique trace-preserving conditional expectation $E_{M_{0}}: M_{1} \rightarrow M_{0}$ determined by $\operatorname{tr}_{1}(x y)=\operatorname{tr}_{0}\left(E_{M_{0}}(x) y\right)$ for all $x \in M_{1}$ and $y \in$ $M_{0}$, i.e. $E_{M_{0}}$ is the (Banach) adjoint of the inclusion of preduals $\left(M_{0}\right)_{*} \rightarrow\left(M_{1}\right)_{*}$. The conditional expectation satisfies $e_{1}(x \Omega)=E_{M_{0}}(x) \Omega$ for all $x \in M_{1}$.

The following proposition is straightforward.

Proposition 2.2. The following are equivalent for a finite subset $B=\{b\} \subset M_{1}$ :

(i) $1=\sum_{b \in B} b e_{1} b^{*}$,

(ii) $x=\sum_{b \in B} b E_{M_{0}}\left(b^{*} x\right)$ for all $x \in M_{1}$, and

(iii) $x=\sum_{b \in B} E_{M_{0}}(x b) b^{*}$ for all $x \in M_{1}$.

Definition 2.3. A Pimsner-Popa basis for $M_{1}$ over $M_{0}$ is a finite subset $B=\{b\} \subset$ $M_{1}$ for which the conditions in Proposition 2.2 hold.

We refer the reader to [23] for the proof of the following result.

Proposition 2.4. The following are equivalent:

(i) there is a Pimsner-Popa basis for $M_{1}$ over $M_{0}$,

(ii) $M_{1} \otimes M_{0} M_{1} \rightarrow M_{2}$ by $x \otimes y \mapsto x e_{1} y$ is an $M_{1}-M_{1}$ bimodule isomorphism, and

(iii) $M_{2}=M_{1} e_{1} M_{1}$.

Remark 2.5. $M_{1} \otimes_{M_{0}} M_{1}$ is a $*$-algebra with multiplication $\left(x_{1} \otimes y_{1}\right)\left(x_{2} \otimes y_{2}\right)=$ $x_{1} \otimes E_{M_{0}}\left(y_{1} x_{2}\right) y_{2}$ and adjoint $(x \otimes y)^{*}=y^{*} \otimes x^{*}$. If there is a Pimsner-Popa basis for $M_{1}$ over $M_{0}$, the sum $\sum_{b \in B} b \otimes b^{*}$ is independent of the choice of Pimsner-Popa basis $B$, as it is the identity. (We will renormalize in Proposition 2.25.) 
Definition 2.6 ([23]). If there is a Pimsner-Popa basis $B=\{b\}$ for $M_{1}$ over $M_{0}$, then we define the (Watatani) index

$$
\left[M_{1}: M_{0}\right]=\sum_{b \in B} b b^{*},
$$

which is independent of the choice of basis.

Definition 2.7. Recall from [21] that $M_{2}$ has a canonical faithful, normal, semifinite trace $\operatorname{Tr}_{2}$ which is the extension of the map $x e_{1} y \mapsto \operatorname{tr}_{1}(x y)$ for $x, y \in M_{1}$.

Definition 2.8. An inclusion $M_{0} \subset\left(M_{1}, \operatorname{tr}_{1}\right)$ of finite von Neumann algebras is called Markov if it satisfies the Markov property:

(1) $\operatorname{Tr}_{2}$ is finite with $\left.\operatorname{Tr}_{2}(1)^{-1} \operatorname{Tr}_{2}\right|_{M_{1}}=\operatorname{tr}_{1}$.

A Markov inclusion is called strongly Markov if

(2) there is a Pimsner-Popa basis for $M_{1}$ over $M_{0}$.

Remark 2.9. Markov inclusions have been studied by Jolissaint [7], Pimsner and Popa [18] and [21], and more. Jolissaint [7] showed that condition (1) implies condition (2) when the centers are atomic and the inclusion is connected, i.e. $Z\left(M_{0}\right) \cap$ $Z\left(M_{1}\right)=M_{1}^{\prime} \cap M_{0}$ is one dimensional. It is unknown to the authors at this point whether condition (1) implies condition (2) for connected inclusions with diffuse centers.

The adjective "strongly" in the term "strongly Markov" comes from Definition 3.6 in [1], where they define the notion of "fortement d'indice fini" for a conditional expectation. This notion translates as the existence of a finite Pimsner-Popa basis.

Remark 2.10. Recall from [21] that $\operatorname{Tr}_{2}(1)^{-1} \operatorname{Tr}_{2}$ extends $\operatorname{tr}_{1}$ if and only if $\operatorname{Tr}_{2}(1)=$ $\left[M_{1}: M_{0}\right] \in[1, \infty)$.

Examples 2.11. (1) A finite Jones index inclusion of $\mathrm{II}_{1}$-factors with the unique trace is strongly Markov, and the Watatani index is equal to the Jones index.

(2) A connected, unital inclusion of finite dimensional $C^{*}$-algebras with the Markov trace is strongly Markov, and the index is equal to $\left\|\Lambda^{T} \Lambda\right\|$ where $\Lambda$ is the bipartite adjacency matrix for the Bratteli diagram of the inclusion.

Suppose $M_{0} \subset\left(M_{1}, \operatorname{tr}_{1}\right)$ is strongly Markov. Then $M_{2}$ is finite and $\operatorname{tr}_{2}=$ $\left[M_{1}: M_{0}\right]^{-1} \operatorname{Tr}_{2}$ extends $\operatorname{tr}_{1}$, so we may iterate the basic construction for $M_{1} \subset$ $\left(M_{2}, \operatorname{tr}_{2}\right)$. Let $M_{3}=\left\langle M_{2}, e_{2}\right\rangle \subset B\left(L^{2}\left(M_{2}, \operatorname{tr}_{2}\right)\right)$, where $e_{2}$ is the Jones projection with range $L^{2}\left(M_{1}, \operatorname{tr}_{1}\right)$. Let $\operatorname{Tr}_{3}$ be the canonical faithful, normal, semifinite trace on $M_{3}$ (see Definition 2.7). The following lemma is straightforward. 
Lemma 2.12. (1) The conditional expectation $E_{M_{1}}: M_{2} \rightarrow M_{1}$ is given by

$$
E_{M_{1}}\left(x e_{1} y\right)=x y,
$$

(2) $e_{1} e_{2} e_{1}=\left[M_{1}: M_{0}\right]^{-1} e_{1}$ and $e_{2} e_{1} e_{2}=\left[M_{1}: M_{0}\right]^{-1} e_{2}$, and

(3) if B is a Pimsner-Popa basisfor $M_{1}$ over $M_{0}$, then $\left\{\left[M_{1}: M_{0}\right]^{1 / 2} b e_{1} \mid b \in B\right\}$ is a Pimsner-Popa basis for $M_{2}$ over $M_{1}$.

Theorem 2.13. $M_{1} \subset\left(M_{2}, \operatorname{tr}_{2}\right)$ is strongly Markov and $\left[M_{2}: M_{1}\right]=\left[M_{1}: M_{0}\right]$.

Proof. Note $M_{3}=M_{2} e_{2} M_{2}$ by Proposition 2.4 and Lemma 2.12, so the canonical trace $\operatorname{Tr}_{3}$ on $M_{3}$ is finite. By Definition 2.7 and Lemma 2.12, if $x \in M_{2}$,

$$
\begin{aligned}
\operatorname{Tr}_{3}(x) & =\left[M_{1}: M_{0}\right] \sum_{b \in B} \operatorname{Tr}_{3}\left(x b e_{1} e_{2} e_{1} b^{*}\right)=\left[M_{1}: M_{0}\right] \sum_{b \in B} \operatorname{tr}_{2}\left(x b e_{1} b^{*}\right) \\
& =\left[M_{1}: M_{0}\right] \operatorname{tr}_{2}(x) .
\end{aligned}
$$

Hence $\left[M_{2}: M_{1}\right]=\operatorname{Tr}_{3}(1)=\left[M_{1}: M_{0}\right]$, and $\operatorname{tr}_{3}=\left[M_{1}: M_{0}\right]^{-1} \operatorname{Tr}_{3}$ extends $\operatorname{tr}_{2}$.

Definition 2.14. Suppose $P \subset B\left(L^{2}\left(M_{1}, \operatorname{tr}_{1}\right)\right)$ is a von Neumann algebra containing $M_{1}, \operatorname{tr}_{P}$ is a trace on $P$ extending $\operatorname{tr}_{1}$, and $p$ is a projection in $P$. We say the inclusion $M_{0} \subset M_{1} \subset\left(P, \operatorname{tr}_{P}, p\right)$ is standard if there is an isomorphism of von Neumann algebras $\varphi: P \rightarrow M_{2}$ such that $\left.\varphi\right|_{M_{1}}=\operatorname{id}_{M_{1}}, \operatorname{tr}_{P}=\operatorname{tr}_{2} \circ \varphi$, and $\varphi(p)=e_{1}$.

The following lemma, which is an alteration of Lemma 5.8 of [7] and uses ideas from Lemma 5.3.1 in [12], allows us to identify when inclusions are standard.

Lemma 2.15. Suppose $M_{0} \subset M_{1} \subset\left(P, \operatorname{tr}_{P}, p\right)$ such that

(1) $p m p=E_{M_{0}}(m) p$ for all $m \in M_{1}$, and

(2) $E_{M_{1}}(p)=\left[M_{1}: M_{0}\right]^{-1}$.

Then $\psi: M_{1} \otimes_{M_{0}} M_{1} \rightarrow M_{1} p M_{1}$ by $x \otimes y \mapsto x p y$ is an $M_{1}$-bilinear isomorphism of $*$-algebras. Hence $\varphi: M_{1} e_{1} M_{1} \rightarrow M_{1} p M_{1}$ by $x e_{1} y \mapsto x p y$ is an isomorphism of $*$-algebras. Moreover, if

(3) $P=M_{1} p M_{1}$,

then $M_{0} \subset M_{1} \subset\left(P, \operatorname{tr}_{P}, p\right)$ is standard via $\varphi$. Conversely, if $M_{0} \subset M_{1} \subset$ $\left(P, \operatorname{tr}_{P}, p\right)$ is standard, then (1), (2), and (3) hold.

Proof. First, note that $p x=x p$ for all $x \in M_{0}$ by (1), and the map $M_{1} \rightarrow M_{1} p$ by $y \mapsto y p$ is injective by (2). Clearly $\psi$ is surjective and preserves the $*$-algebra structure. Suppose

$$
\psi\left(\sum_{i=1}^{k} x_{i} \otimes y_{i}\right)=\sum_{i=1}^{k} x_{i} p y_{i}=0
$$


Then for all $x, y \in M_{1}$,

$$
p x\left(\sum_{i=1}^{k} x_{i} p y_{i}\right) y p=\left(\sum_{i=1}^{k} E_{M_{0}}\left(x x_{i}\right) E_{M_{0}}\left(y_{i} y\right)\right) p=0,
$$

which implies $\sum_{i=1}^{k} E_{M_{0}}\left(x x_{i}\right) E_{M_{0}}\left(y_{i} y\right)=0$. If $B=\{b\}$ is a Pimsner-Popa basis for $M_{1}$ over $M_{0}$, by Remark 2.5,

$$
\begin{aligned}
\sum_{i=1}^{k} x_{i} \otimes y_{i} & =\sum_{a \in B} a \otimes a^{*}\left(\sum_{i=1}^{k} x_{i} \otimes y_{i}\right) \sum_{b \in B} b \otimes b^{*} \\
& =\sum_{a, b \in B} \sum_{i=1}^{k} a \otimes E_{M_{0}}\left(a^{*} x_{i}\right) E_{M_{0}}\left(y_{i} b\right) b^{*}=0 .
\end{aligned}
$$

The remaining claims follow as in [7].

2.2. The Jones tower and tensor products. We give the background necessary to define the canonical planar *-algebra associated to a Markov inclusion and to prove its uniqueness. Many facts stated without proof in Subsection 2.6 rely on the results of this subsection. In particular, the multistep basic construction described in this subsection helps us understand tangles which cap off on the left (see Proposition 2.47), which are crucial to the proof of Theorem 4.1, the main result of this paper.

For the rest of this section, let $M_{0} \subset\left(M_{1}, \operatorname{tr}_{1}\right)$ be a strongly Markov inclusion of finite von Neumann algebras, and set $d=\left[M_{1}: M_{0}\right]^{1 / 2}$. For $n \in \mathbb{N}$, inductively define the basic construction

$$
M_{n+1}=\left\langle M_{n}, e_{n}\right\rangle=M_{n} e_{n} M_{n}=J_{n} M_{n-1}^{\prime} J_{n} \subset B\left(L^{2}\left(M_{n}, \operatorname{tr}_{n}\right)\right)
$$

with canonical trace $\operatorname{tr}_{n+1}$ extending $\operatorname{tr}_{n}$ and satisfying $\operatorname{tr}_{n+1}\left(x e_{n}\right)=d^{-2} \operatorname{tr}_{n}(x)$ for all $x \in M_{n}$ where $e_{n} \in B\left(L^{2}\left(M_{n}, \operatorname{tr}_{n}\right)\right)$ is the Jones projection with range $L^{2}\left(M_{n-1}, \operatorname{tr}_{n-1}\right)$. For $n \in \mathbb{N}$, set $E_{n}=d e_{n}$.

Fact 2.16. The $E_{i}$ 's satisfy the Temperley-Lieb relations

(i) $E_{i}^{2}=d E_{i}=d E_{i}^{*}$,

(ii) $E_{i} E_{j}=E_{j} E_{i}$ for $|i-j|>1$, and

(iii) $E_{i} E_{i \pm 1} E_{i}=E_{i}$.

Proposition 2.17. Suppose $N \subset\left(M, \operatorname{tr}_{M}\right)$ and $M \subset\left(P, \operatorname{tr}_{P}\right)$ such that $\left.\operatorname{tr}_{P}\right|_{M}=$ $\operatorname{tr}_{M}$. Suppose $A=\{a\}$ is a Pimsner-Popa basis for $P$ over $M$ and $B=\{b\}$ is a Pimsner-Popa basis for $M$ over $N$. Then

(1) $A B=\{a b \mid a \in A$ and $b \in B\}$ is a Pimsner-Popa basis for $P$ over $N$,

(2) $[P: N]=[P: M][M: N]$, and 
(3) $\sum_{b \in B} b e_{N}^{P} b^{*}=e_{M}^{P} \in B\left(L^{2}\left(P, \operatorname{tr}_{P}\right)\right)$, where $e_{N}^{P}$ is the projection $L^{2}\left(P, \operatorname{tr}_{P}\right) \rightarrow$ $L^{2}\left(N, \operatorname{tr}_{N}\right)$ and $e_{M}^{P}$ is the projection $L^{2}\left(P, \operatorname{tr}_{P}\right) \rightarrow L^{2}\left(M, \operatorname{tr}_{M}\right)$.

Proof. (1) For all $x \in P$,

$$
\begin{aligned}
\sum_{a b \in A B} a b E_{N}^{P}\left(b^{*} a^{*} x\right) & =\sum_{a, b} a b E_{N}^{M}\left(E_{M}^{P}\left(b^{*} a^{*} x\right)\right)=\sum_{a, b} a b E_{N}^{M}\left(b^{*} E_{M}^{P}\left(a^{*} x\right)\right) \\
& =\sum_{a} a E_{M}^{P}\left(a^{*} x\right)=x .
\end{aligned}
$$

(2) Immediate from (1).

(3) If $p \in P$ and $\Omega \in L^{2}\left(P, \operatorname{tr}_{P}\right)$ is the image of $1 \in P$, then

$$
\begin{aligned}
\sum_{b \in B} b e_{N}^{P} b^{*} p \Omega & =\sum_{b \in B} b E_{N}^{P}\left(b^{*} p\right) \Omega=\sum_{b \in B} b E_{N}^{M}\left(b^{*}\left(E_{M}^{P}(p)\right) \Omega\right. \\
& =E_{M}^{P}(p) \Omega=e_{M}^{P} p \Omega .
\end{aligned}
$$

Corollary 2.18. $M_{k} \subset\left(M_{n}, \operatorname{tr}_{n}\right)$ is strongly Markov for all $0 \leq k \leq n$.

The following technical lemma will be used to define the multistep basic construction of [19], [7], and [3] in Proposition 2.20.

Lemma 2.19. For all $0 \leq k \leq n$, define the following element of $M_{k+n}$ (see Remark 2.45):

$f_{n-k}^{n}=d^{k(k-1)}\left(e_{n} e_{n-1} \ldots e_{n-k+1}\right)\left(e_{n+1} e_{n} \ldots e_{n-k+2}\right) \ldots\left(e_{n+k-1} e_{n+k-2} \ldots e_{n}\right)$.

If $0 \leq j \leq k \leq n$ and $B$ is a Pimsner-Popa basis for $M_{n-j}$ over $M_{n-k}$, then $\sum_{b \in B} b f_{n-k}^{n} b^{*}=f_{n-j}^{n}$.

Proof. For $j+1 \leq i \leq k$, let $A_{i}$ be a Pimsner-Popa basis for $M_{n-i+1}$ over $M_{n-i}$. Then $A=A_{j+1} \ldots A_{k}$ is a Pimsner-Popa basis for $M_{n-j}$ over $M_{n-k}$ by Proposition 2.17 , and

$$
\begin{aligned}
& \sum_{\substack{a_{i} \in A_{i} \\
j+1 \leq i \leq k}} a_{j+1} \ldots a_{k} f_{n-k}^{n} a_{k}^{*} \ldots a_{j+1}^{*}=\sum_{\substack{a_{i} \in A_{i} \\
j+1 \leq i \leq k-1}} a_{j+1} \ldots a_{k-1} f_{n-k+1}^{n} a_{k-1}^{*} \ldots a_{j+1}^{*} \\
& =\cdots=\sum_{a_{j+1} \in A_{j+1}} a_{j+1} f_{n-j-1}^{n} a_{j+1}^{*}=f_{n-j}^{n} .
\end{aligned}
$$

Let $B$ be another Pimsner-Popa basis for $M_{n-j}$ over $M_{n-k}$ and let us define $U \in$ $\operatorname{Mat}_{|A| \times|B|}\left(M_{n-k}\right)$ by $U_{a, b}=E_{M_{n-k}}^{M_{n-j}}\left(a^{*} b\right)$. If we consider $A$ as a row vector in $\operatorname{Mat}_{1 \times|A|}\left(M_{n-j}\right)$, then $B=A U$ and $A=B U^{*}$. For $\ell \in \mathbb{N}$, let $F_{\ell}=f_{n-k}^{n} I_{\ell} \in$ 
$\operatorname{Mat}_{\ell \times \ell}\left(M_{n+k}\right)$, i.e. $F_{\ell}$ is the $\ell \times \ell$ diagonal matrix with all diagonal entries equal to $f_{n-k}^{n}$. Then since $f_{n-k}^{n}$ commutes with $M_{n-k}$, we have

$$
\begin{aligned}
\sum_{b \in B} b f_{n-k}^{n} b^{*} & =B F_{|B|} B^{*}=A U F_{|B|} U^{*} A^{*}=A U U^{*} F_{|A|} A^{*}=A F_{|A|} A^{*} \\
& =\sum_{a \in A} a f_{n-k}^{n} a^{*}=f_{n-j}^{n} .
\end{aligned}
$$

Proposition 2.20 (Multistep basic construction). The inclusion $M_{n-k} \subset M_{n} \subset$ $\left(M_{n+k}, \operatorname{tr}_{n+k}, f_{n-k}^{n}\right)$ is standard.

Proof. Let $B$ be a Pimsner-Popa basis for $M_{n}$ over $M_{n-k}$. Then by Lemma 2.19, $\sum_{b \in B} b f_{n-k}^{n} b^{*}=1$, so $M_{n} f_{n-k}^{n} M_{n}=M_{n+k}$. It is straightforward to check $f_{n-k}^{n} x f_{n-k}^{n-k}=E_{M_{n-k}}(x) f_{n-k}^{n}$ for all $x \in M_{n}$ and $E_{M_{n}}\left(f_{n-k}^{n}\right)=d^{-2 k}$, and the result follows by Lemma 2.15 .

Remark 2.21. Note that $L^{2}\left(M_{n}, \operatorname{tr}_{n}\right)$ has left and right actions of $M_{0}, \ldots, M_{2 n}$, where as usual, the right action of $M_{i}$ is the left action of $J_{n} M_{i} J_{n} \cong M_{i}^{\text {op }}$. Note that $M_{i}^{\prime}=J_{n} M_{2 n-i} J_{n}$, so we define a canonical trace on $M_{i}^{\prime} \cap B\left(L^{2}\left(M_{n}, \operatorname{tr}_{n}\right)\right)$ by $\operatorname{tr}_{i}^{\prime}(x)=\operatorname{tr}_{2 n-i}\left(J_{n} x^{*} J_{n}\right)$ for all $x \in M_{i}^{\prime} \cap B\left(L^{2}\left(M_{n}, \operatorname{tr}_{n}\right)\right)$.

Proposition 2.22 (Shifts). For all $0 \leq k \leq n$, there is a canonical isomorphism $M_{k}^{\prime} \cap M_{n} \cong M_{k+2}^{\prime} \cap M_{n+2}$.

Proof. On $B\left(L^{2}\left(M_{n}, \operatorname{tr}_{n}\right)\right)$, the map $x \mapsto J_{n} x^{*} J_{n}$ gives an anti-isomorphism $M_{k}^{\prime} \cap$ $M_{n} \cong M_{n}^{\prime} \cap M_{2 n-k}$. On $B\left(L^{2}\left(M_{n+1}, \operatorname{tr}_{n+1}\right)\right)$, the map $x \mapsto J_{n} x^{*} J_{n}$ gives an anti-isomorphism $M_{n}^{\prime} \cap M_{2 n-k} \cong M_{k+2}^{\prime} \cap M_{n+2}$.

Proposition 2.23. The canonical trace-preserving conditional expectation $M_{n+k} \rightarrow$ $M_{n+k-i}$ is given by $x f_{n-k}^{n} y \mapsto d^{-2 i} x f_{n-k+i}^{n} y$ where $x, y \in M_{n}$. The canonical trace-preserving conditional expectation $M_{n-k}^{\prime}=J_{n} M_{n+k} J_{n} \rightarrow J_{n} M_{n+k-i} J_{n}=$ $M_{n-k+i}^{\prime}$ is given by the same formula, only with $x, y \in M_{n}^{\prime}=J_{n} M_{n} J_{n}$.

Proof. We prove the first statement, as the second is similar. By the Markov property, for all $x, y \in M_{n}$,

$$
\operatorname{tr}_{n+k}\left(x f_{n-k}^{n} y\right)=d^{-2 k} \operatorname{tr}_{n}(x y)=d^{-2 i} \operatorname{tr}_{n+k-i}\left(x f_{n-k+i}^{n} y\right),
$$

so the map is trace-preserving. Now $M_{n+k-i}$-bilinearity follows from the following two facts:

(i) for all $1 \leq i \leq k, M_{n-k} \subset M_{n-k+i}$, so $f_{n-k+i}^{n} f_{n-k}^{n}=f_{n-k}^{n}$, and

(ii) $E_{M_{n+k-i}}^{M_{n+k}}\left(f_{n-k}^{n}\right)=d^{-2 i} f_{n-k+i}^{n}$. 
We can now strengthen Proposition 2.7 from [3], versions of which also appear in [4]. This is the main proposition describing left-capping tangles.

Proposition 2.24. Let $0 \leq k \leq \ell \leq n$, and let $B$ be a Pimsner-Popa basis for $M_{\ell}$ over $M_{k}$. The conditional expectation $E_{M_{\ell}^{\prime}}^{M_{k}^{\prime}}:\left(M_{k}^{\prime} \cap B\left(L^{2}\left(M_{n}, \operatorname{tr}_{n}\right)\right), \operatorname{tr}_{k}^{\prime}\right) \rightarrow$ $\left(M_{\ell}^{\prime} \cap B\left(L^{2}\left(M_{n}, \operatorname{tr}_{n}\right)\right), \operatorname{tr}_{\ell}^{\prime}\right)$ is given by

$$
E_{M_{\ell}^{\prime}}^{M_{k}^{\prime}}(x)=\frac{1}{d^{2(\ell-k)}} \sum_{b \in B} b x b^{*} .
$$

In particular, this map is independent of $n$ and the choice of basis.

Proof. The result follows from Lemma 2.19 and Proposition 2.23, since for $x, y \in$ $J_{n} M_{n} J_{n} \subset M_{\ell}^{\prime}$,

$$
\sum_{b \in B} b x f_{k}^{n} y b^{*}=\sum_{b \in B} x b f_{k}^{n} b^{*} y=x f_{\ell}^{n} y .
$$

To define our planar $*$-algebra in Subsection 2.3, we need the following fact, which follows from Proposition 2.4 and a simple induction argument.

Proposition 2.25. For $k \in \mathbb{N}$, let $v_{k}=E_{k} E_{k-1} \ldots E_{1}$. For all $n \in \mathbb{N}$, there are isomorphisms of $M_{1}-M_{1}$ bimodules

$$
\begin{aligned}
\theta_{n}: \quad \bigotimes_{M_{0}}^{n} M_{1} & \longrightarrow \quad \begin{array}{c}
M_{n}, \\
x_{1} \otimes \cdots \otimes x_{n}
\end{array} x_{1} v_{1} x_{2} v_{2} \ldots v_{n-1} x_{n} .
\end{aligned}
$$

Remark 2.26. Recall that $L^{2}\left(M_{n}, \operatorname{tr}_{n}\right)$ is the completion of $M_{n}$ with inner product $\langle x, y\rangle=\operatorname{tr}_{n}\left(y^{*} x\right)$. As usual, $\theta_{n}$ gives an isomorphism of Hilbert-bimodules

$$
\bigotimes_{M_{0}}^{n} L^{2}\left(M_{1}, \operatorname{tr}_{1}\right) \longrightarrow L^{2}\left(M_{n}, \operatorname{tr}_{n}\right)
$$

where the tensor product on the left is Connes' relative tensor product with inner product given inductively by

$$
\begin{aligned}
\left\langle x_{1} \otimes u, y_{1} \otimes v\right\rangle_{n} & =\left\langle E_{M_{0}}\left(y_{1}^{*} x_{1}\right) u, v\right\rangle_{n-1}, \\
\left\langle u \otimes x_{n}, v \otimes y_{n}\right\rangle_{n} & =\left\langle u, v E_{M_{0}}\left(y_{n} x_{n}^{*}\right)\right\rangle_{n-1} .
\end{aligned}
$$

The following operators will be useful in the definition of the rotation operators in Subsections 2.4 and 2.5. 
Definition 2.27. Given $x \in M_{1}$, we get left and right multiplication operators

$$
L(x), R(x): \bigotimes_{M_{0}}^{n} L^{2}\left(M_{1}, \operatorname{tr}_{1}\right) \longrightarrow \bigotimes_{M_{0}}^{n} L^{2}\left(M_{1}, \operatorname{tr}_{1}\right)
$$

by $L(x)(v)=x v$ and $R(x)(v)=v x$, and left and right creation operators

$$
L_{x}, R_{x}: \bigotimes_{M_{0}}^{n} L^{2}\left(M_{1}, \operatorname{tr}_{1}\right) \longrightarrow \bigotimes_{M_{0}}^{n+1} L^{2}\left(M_{1}, \operatorname{tr}_{1}\right)
$$

by $L_{x}(v)=x \otimes v$ and $R_{x}(v)=v \otimes x$.

Fact 2.28. For $x, y_{1}, \ldots, y_{n+1} \in M_{1}$, we have

$$
L_{x}^{*}\left(y_{1} \otimes \cdots \otimes y_{n+1}\right)=E_{M_{0}}\left(x^{*} y_{1}\right) y_{2} \otimes \cdots \otimes y_{n+1}
$$

and

$$
R_{x}^{*}\left(y_{1} \otimes \cdots \otimes y_{n+1}\right)=y_{1} \otimes \cdots \otimes y_{n} E_{M_{0}}\left(y_{n+1} x^{*}\right)
$$

The following lemma will be instrumental in defining the action of tangles.

Lemma 2.29. If $A$ is a $\mathbb{C}$-algebra, $V_{1}$ is a right $A$-module, $V_{2}$ is an $A-A$ bimodule, and $V_{3}$ is a left $A$-module, then for each $A$-invariant $v_{2} \in V_{2}$, the map

$$
v_{1} \otimes v_{3} \longmapsto v_{1} \otimes v_{2} \otimes v_{3}
$$

defines a linear map $\varphi_{v_{2}}: V_{1} \otimes_{A} V_{3} \rightarrow V_{1} \otimes_{A} V_{2} \otimes_{A} V_{3}$. Moreover, the map $v \mapsto \varphi_{v}$ on $A^{\prime} \cap V_{2}=\left\{v \in V_{2} \mid a v=v a\right.$ for all $\left.a \in A\right\}$ is $\mathbb{C}$-linear.

Proof. Middle $A$-linearity is satisfied as $v_{2}$ is $A$-invariant.

Remark 2.30. This lemma gives an alternate proof that the map $E_{M_{1}^{\prime}}^{M_{0}^{\prime}}$ is well defined in Proposition 2.24. By Remark 2.5, $d^{-2} \sum_{b \in B} b \otimes b^{*}$ is independent of the choice of Pimsner-Popa basis $B$, so the composite map

$$
x \longmapsto \varphi_{x} \longmapsto \varphi_{x}\left(d^{-2} \sum_{b \in B} b \otimes b^{*}\right)=d^{-2} \sum_{b \in B} b \otimes x \otimes b^{*} \longmapsto d^{-2} \sum_{b \in B} b x b^{*}
$$

on $M_{0}^{\prime} \cap B\left(L^{2}\left(M_{n}, \operatorname{tr}_{n}\right)\right)$ is independent of the choice. Moreover, the result is $M_{1}$-invariant, since for any unitary $u \in M_{1},\{u b \mid b \in B\}$ is another Pimsner-Popa basis for $M_{1}$ over $M_{0}$. 
2.3. Definition of the canonical planar $*$-algebra. The definition of a planar $*$-algebra has evolved since its inception in [9]. We use the definition of [11] (see also [17]), but we do not reproduce it here.

In [9], it was shown how to endow the tower of relative commutants of an extremal, finite index $\mathrm{II}_{1}$-subfactor with the structure of a subfactor planar algebra, i.e. a planar *-algebra $Q_{\bullet}=\left\{Q_{n, \pm}\right\}$ with $\operatorname{dim}\left(Q_{n, \pm}\right)<\infty$ for all $n \geq 0$ which is

- spherical, i.e $\operatorname{dim}\left(Q_{0, \pm}\right)=1$ and any fully labelled 0 -tangle is invariant under spherical isotopy. This implies shaded and unshaded contractible loops count for the same multiplicative factor of $d$, called the modulus of $Q_{\bullet}$, and

- positive definite, i.e. the bilinear form on $Q_{n, \pm}$ given by $\langle a, b\rangle=d^{-n} \operatorname{tr}\left(b^{*} a\right)$ is positive definite.

The only essential ingredient to the construction of [9] is a Pimsner-Popa basis, so the same construction applies to a strongly Markov inclusion $M_{0} \subset\left(M_{1}, \operatorname{tr}_{1}\right)$. As we do not require the algebras to be factors or the inclusion to be extremal, the resulting planar algebra need not be spherical nor positive-definite nor have finite dimensional $n$-box spaces.

Below, we define a planar $*$-algebra structure on the vector spaces $P_{n, \pm}(n \geq 0)$ given by $P_{n,+}=\theta_{n}^{-1}\left(M_{0}^{\prime} \cap M_{n}\right)$ and $P_{n,-}=\theta_{n}^{-1}\left(M_{1}^{\prime} \cap M_{n+1}\right)$. This planar algebra is independent of any choices, so we call it the canonical planar $*$-algebra associated to $M_{0} \subset\left(M_{1}, \operatorname{tr}_{1}\right)$.

We define the action of a planar tangle in standard form:

(1) all the input and output disks are horizontal rectangles with all strings (that are not closed loops) emanating from the top edges of the rectangles,

(2) all the input disks are in disjoint horizontal bands and all maxima and minima of strings are at different vertical levels, and not in the horizontal bands defined by the input disks, and

(3) the distinguished (starred) intervals of all the disks are at the left edges of the rectangles. (In the sequel, we will assume this convention and omit the $*$ 's.)

We do not provide the proof of isotopy invariance, i.e. that the action is independent of the choice of standard form, as this proof is identical to that in [9]. However, in Subsection 2.4, we provide Burns' elegant proof that the rotation operator is welldefined.

Suppose we have a $(k, \pm)$-tangle $T$ in standard form with $s$ input rectangles, and input rectangle $j$ has $2 r_{j}$ strings emanating from the top. We define the action of $T$ on an $s$-tuple $\xi=\left(\xi_{1}, \ldots, \xi_{s}\right)$ where $\xi_{j} \in P_{r_{j}, \pm_{j}}$ and $\pm_{j}= \pm$ if the region just below input rectangle $j$ is unshaded or shaded respectively.

We read the action of $T$ on $\xi$ by sliding a horizontal line through the tangle from bottom to top. For a fixed vertical $y$-value, off the input disks' horizontal bands and away from the relative extrema of the strings, the horizontal line will meet $n_{y}$ shaded regions from left to right. One should think of the shaded regions along this line as elements of $M_{1}$ and the unshaded regions between shaded regions as the symbols 
$\otimes_{M_{0}}$. Near the top, the line will meet $k$ or $k+1$ shaded regions depending on whether the left-most region of $T$ is unshaded or shaded respectively. We illustrate a typical $(3,+)$-tangle with the horizontal line about half way through its travel:

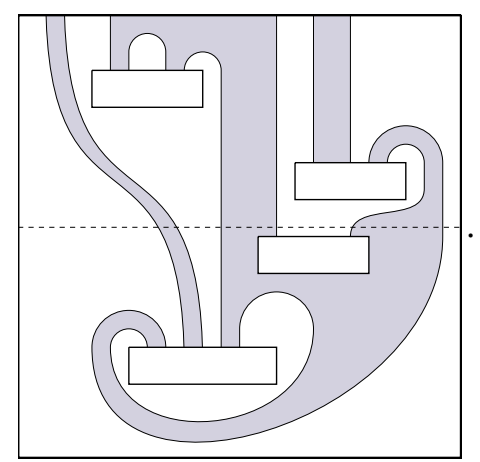

For each $y$ coordinate of the horizontal line, one reads off an $M_{i}$-invariant element $\eta_{y} \in \bigotimes_{M_{0}}^{n_{y}} M_{1}$, where $i=0$ if $T$ is a $(k,+)$-tangle and $i=1$ if $T$ is a $(k,-)$-tangle.

The element $\eta_{y}$ begins as $1 \in M_{i}$ near the bottom, and it remains constant as long as the horizontal line meets neither maxima, minima, nor rectangles. If the horizontal line passes input rectangle $j$ for which exactly $t$ shaded regions sit to the left, then we insert $\xi_{j}$ into $\eta_{y}$ as in Figure 1 by applying Lemma 2.29 with $v_{2}=\xi_{j}$,

$$
V_{1}=\bigotimes_{M_{0}}^{t} M_{1}, \quad V_{2}=P_{r_{j}, \pm_{j}}, \text { and } V_{3}=\bigotimes_{M_{0}}^{n_{y}-t} M_{1}
$$

Note that $V_{1}, V_{3}$ are considered as $M_{\ell}$-modules and $P_{r_{j}, \pm_{j}}$ is an $M_{\ell}-M_{\ell}$ bimodule, where $\ell=0$ if $\pm_{j}=+$ and $\ell=1$ if $\pm_{j}=-$. Note that inserting $\xi_{j}$ into $\eta_{y}$ gives an $M_{i}$-invariant vector.

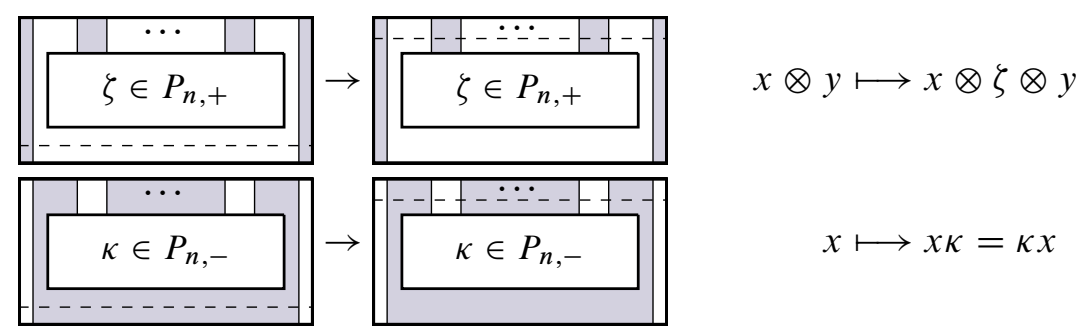

Figure 1. Inserting central vectors.

As the horizontal line passes a maximum or minimum, $\eta_{y}$ changes according to Figure 2 where the changes indicated on the tensors are to be inserted into the position indicated by the shaded regions on the horizontal (dashed) line. With the exception 
of one case, each of these maps is a $\left(M_{1}-M_{1}\right)$-bimodule map, so it will preserve $M_{i}$-invariant elements. The remaining case to consider is when the left-most or rightmost shaded region is capped off by applying the third map pictured above, which is a $\left(M_{0}-M_{0}\right)$-bimodule map. But this will only occur when the distinguished (starred) interval of the external disk meets an unshaded region, so $i$ would have to be 0 from the beginning.
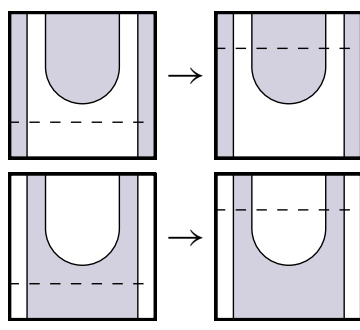

$$
x \otimes y \longmapsto x \otimes 1 \otimes y
$$

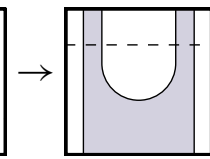

$$
x \longmapsto d^{-1} \sum_{b \in B} x b \otimes b^{*}=d^{-1} \sum_{b \in B} b \otimes b^{*} x
$$

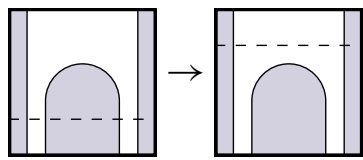

$x \otimes y \otimes z \longmapsto d x E_{M_{0}}(y) \otimes z=d x \otimes E_{M_{0}}(y) z$

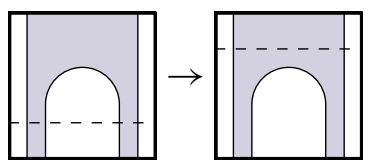

$$
x \otimes y \longmapsto x y
$$

Figure 2. Reading maxima and minima of planar tangles in standard form.

The action of the tangle on $\xi$ is the element $\eta_{y} \in P_{k, \pm}$ read for horizontal lines sufficiently close to the top. The $*$-structure is the same as that of [9].

Example 2.31. To calculate

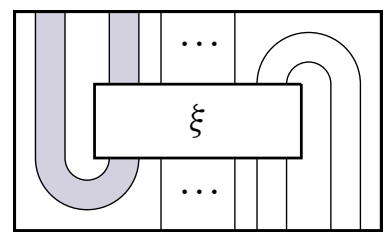

for

$$
\xi=\sum_{i=1}^{k} x_{1}^{i} \otimes \cdots \otimes x_{n}^{i} \in \theta_{n}^{-1}\left(M_{0}^{\prime} \cap M_{n}\right),
$$

we first isotope the tangle into a standard form. The horizontal line travels upward 
as shown:

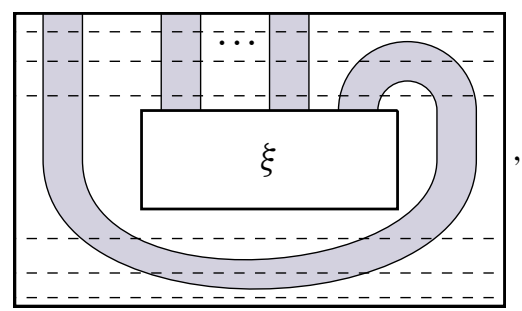

which we read as

$$
\begin{aligned}
1_{\mathbb{C}} & \mapsto 1_{M} \mapsto d^{-1} \sum_{b \in B} b \otimes b^{*} \mapsto d^{-1} \sum_{b \in B} b \otimes \xi \otimes b^{*} \\
& \mapsto d^{-1} \sum_{b \in B} \sum_{i=1}^{k} b \otimes x_{1}^{i} \otimes \cdots \otimes x_{n-1}^{i} \otimes x_{n}^{i} b^{*} \\
& \mapsto \sum_{b \in B} \sum_{i=1}^{k} b \otimes x_{1}^{i} \otimes \cdots \otimes x_{n-1}^{i} E_{M_{0}}\left(x_{n}^{i} b^{*}\right),
\end{aligned}
$$

the last line giving the output of the tangle applied to $\xi$.

2.4. Burns' treatment of the rotation operator on $\boldsymbol{P}_{\boldsymbol{n},+}$. The key to showing that the $P_{n, \pm}$ 's define a planar algebra is isotopy invariance, which relies on the existence of the rotation on $P_{n, \pm}$. A particularly elegant treatment of this is due to Michael Burns, but it only appears in his thesis [4], so we include a proof below for the reader's convenience.

Definition 2.32. Let $B$ be a Pimsner-Popa basis of $M_{1}$ over $M_{0}$. For all $x=$ $x_{1} \otimes \cdots \otimes x_{n} \in \bigotimes_{M_{0}}^{n} M_{1}$, define

$$
\rho(x)=\sum_{b \in B} L_{b} R_{b}^{*}(x)=\sum_{b \in B} b \otimes x_{1} \otimes \cdots \otimes x_{n-1} E_{M_{0}}\left(x_{n} b^{*}\right)
$$

(see Example 2.31).

Proposition 2.33. The map $\rho$ preserves $P_{n,+}$, and its restriction to $P_{n,+}$ is independent of the choice of $B$.

Proof. Middle linearity is respected by $\rho$, so it is well defined, though it may depend on $B$. By Lemma 2.29 and Remark 2.5, for all $M_{0}$-invariant $x$, the sum $\sum_{b \in B} b \otimes x \otimes b^{*}$ is independent of $B$. We obtain $\rho$ by applying a $\left(M_{0}-M_{0}\right)$-bilinear map which does not involve $B$, so the restriction of $\rho$ is $M_{0}$-invariant and independent of $B$.

Theorem 2.34 ([4]). For $x \in P_{n,+}$ and $y_{1}, \ldots, y_{n} \in M_{1}$,

$$
\left\langle\rho(x), y_{1} \otimes \cdots \otimes y_{n}\right\rangle=\left\langle x, y_{2} \otimes \cdots \otimes y_{n} \otimes y_{1}\right\rangle,
$$

so $\rho^{n}=\mathrm{id}$ on $P_{n,+}$. 


$$
\begin{aligned}
& \text { Proof. As } \rho(x)=\sum_{b \in B} L_{b} R_{b}^{*}(x), \text { we have } \\
& \qquad \begin{aligned}
\left\langle\rho(x), y_{1} \otimes \cdots \otimes y_{n}\right\rangle & =\sum_{b \in B}\left\langle L_{b} R_{b}^{*} x, y_{1} \otimes \cdots \otimes y_{n}\right\rangle \\
& =\sum_{b \in B}\left\langle x, R_{b} L_{b}^{*} y_{1} \otimes \cdots \otimes y_{n}\right\rangle \\
& =\sum_{b \in B}\left\langle x, E_{M_{0}}\left(b^{*} y_{1}\right) y_{2} \otimes \cdots \otimes y_{n} \otimes b\right\rangle \\
& =\sum_{b \in B}\left\langle E_{M_{0}}\left(b^{*} y_{1}\right)^{*} x, y_{2} \otimes \cdots \otimes y_{n} \otimes b\right\rangle \\
& =\sum_{b \in B}\left\langle x E_{M_{0}}\left(b^{*} y_{1}\right)^{*}, y_{2} \otimes \cdots \otimes y_{n} \otimes b\right\rangle \\
& =\sum_{b \in B}\left\langle x, y_{2} \otimes \cdots \otimes y_{n} \otimes b E_{M_{0}}\left(b^{*} y_{1}\right)\right\rangle \\
& =\left\langle x, y_{2} \otimes \cdots \otimes y_{n} \otimes y_{1}\right\rangle .
\end{aligned}
\end{aligned}
$$

Corollary 2.35. The rotation

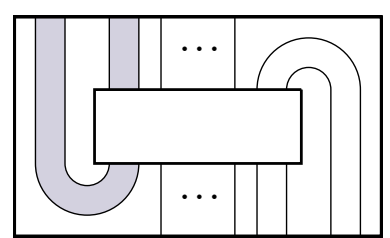

on $P_{n,+}$ is well defined.

2.5. The rotation on $\boldsymbol{P}_{\boldsymbol{n},-}$. We mimic Burns' treatment of the rotation on $P_{n,+}$ to define the rotation on $P_{n,-}$.

Definition 2.36. Let $B$ be a Pimsner-Popa basis of $M_{1}$ over $M_{0}$. For

$$
x=x_{1} \otimes \cdots \otimes x_{n+1} \in \bigotimes_{M_{0}}^{n+1} M_{1},
$$

define $\sigma(x)=\sum_{b \in B} R\left(b^{*}\right) R_{1}^{*} L_{b}(x)=\sum_{b \in B} b \otimes x_{1} \otimes \cdots \otimes x_{n} E_{M_{0}}\left(x_{n+1}\right) b^{*}$.

Proposition 2.37. The map $\sigma$ preserves $P_{n,-}$, and its restriction to $P_{n,-}$ is independent of the choice of $B$.

Proof. Similar to Proposition 2.33. 
Theorem 2.38. For $x \in P_{n,-}$ and $y_{1}, \ldots, y_{n+1} \in M_{1}$, we have

$$
\left\langle\sigma(x), y_{1} \otimes \cdots \otimes y_{n+1}\right\rangle=\left\langle x, y_{2} \otimes \cdots \otimes y_{n} \otimes y_{n+1} y_{1} \otimes 1\right\rangle .
$$

Proof. Similar to Theorem 2.34.

Corollary 2.39. $\sigma^{n}=\mathrm{id}$ on $P_{n,-}$.

Proof. As $\sigma$ preserves $P_{n,-}$, we repeatedly apply Theorem 2.38 for $x \in P_{n,-}$ to get

$$
\begin{aligned}
\left\langle\sigma^{n}(x), y_{1} \otimes \cdots \otimes y_{n+1}\right\rangle & =\left\langle\sigma^{n-1}(x), y_{2} \otimes \cdots \otimes y_{n} \otimes y_{n+1} y_{1} \otimes 1\right\rangle \\
& =\left\langle\sigma^{n-2}(x), y_{3} \otimes \cdots \otimes y_{n} \otimes y_{n+1} y_{1} \otimes y_{2} \otimes 1\right\rangle \\
& =\cdots=\left\langle x, y_{n+1} y_{1} \otimes y_{2} \otimes \cdots \otimes y_{n} \otimes 1\right\rangle .
\end{aligned}
$$

We then invoke Burns' trick again to get

$$
\begin{aligned}
\left\langle x, y_{n+1} y_{1} \otimes y_{2} \otimes \cdots \otimes y_{n} \otimes 1\right\rangle & =\left\langle y_{n+1}^{*} x, y_{1} \otimes \cdots \otimes y_{n} \otimes 1\right\rangle \\
& =\left\langle x y_{n+1}^{*}, y_{1} \otimes \cdots \otimes y_{n} \otimes 1\right\rangle \\
& =\left\langle x, y_{1} \otimes \cdots \otimes y_{n} \otimes y_{n+1}\right\rangle .
\end{aligned}
$$

Corollary 2.40. The rotation

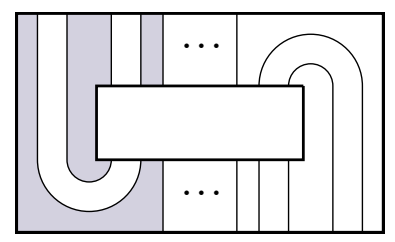

on $P_{n,-}$ is well defined.

2.6. Uniqueness of the canonical planar $*$-algebra. We have the following facts whose proofs are similar to those in [9] and will be omitted (they are straightforward from the results in Subsections 2.1 and 2.2). We shade tangles as much as possible, but sometimes we will not have enough information.

Proposition 2.41 (Multiplication). Suppose $x, y \in M_{n}$ such that

$$
\theta_{n}^{-1}(x)=x_{1} \otimes \cdots \otimes x_{n} \text { and } \theta_{n}^{-1}(y)=y_{1} \otimes \cdots \otimes y_{n}
$$

Then if $n=2 k$ for some $k \in \mathbb{N}$,

$\theta_{n}^{-1}(x y)=x_{1} \otimes \cdots \otimes x_{k} E_{M_{0}}\left(x_{k+1} E_{M_{0}}\left(x_{k+2}(\ldots) y_{k-1}\right) y_{k}\right) \otimes y_{k+1} \otimes \cdots \otimes y_{2 k}$,

while if $n=2 k+1$ for some $k \in \mathbb{N}$,

$\theta_{n}^{-1}(x y)=x_{1} \otimes \cdots \otimes x_{k+1} E_{M_{0}}\left(x_{k+2} E_{M_{0}}\left(x_{k+3}(\ldots) y_{k-1}\right) y_{k}\right) y_{k+1} \otimes \cdots \otimes y_{2 k+1}$. 
Remark 2.42. If $x, y$ as above are in $M_{i}^{\prime} \cap M_{n}$ where $i \in\{0,1\}$, then

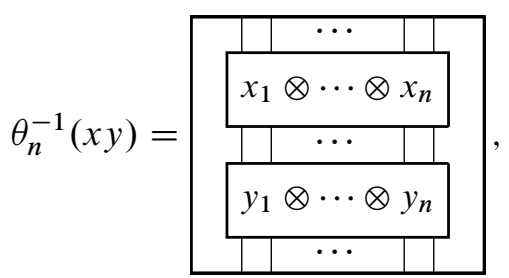

where the shading depends on $i$ and the parity of $n$.

Proposition 2.43 ( $*$-structure). Suppose $x \in M_{n}$ such that $\theta_{n}^{-1}(x)=x_{1} \otimes \cdots \otimes x_{n}$. Then $\theta_{n}^{-1}\left(x^{*}\right)=x_{n}^{*} \otimes \cdots \otimes x_{1}^{*}$.

Proposition 2.44 (Jones projections). For $n \geq 1$, the Jones projection $E_{n} \in P_{n+1,+}$ is given by

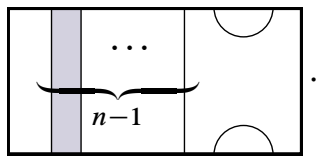

Remark 2.45. The multistep basic construction projection of Proposition 2.20 is given by

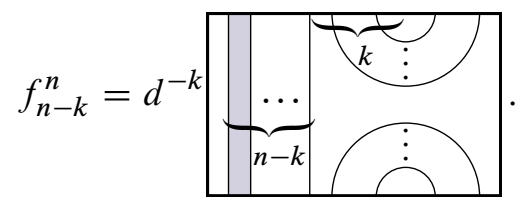

Proposition 2.46 (Inclusions). (1) Let $i_{n}: M_{0}^{\prime} \cap M_{n} \rightarrow M_{0}^{\prime} \cap M_{n+1}$ be the inclusion. Then the inclusion $\theta_{n+1}^{-1} \circ i_{n} \circ \theta_{n}: P_{n, \pm} \rightarrow P_{n+1, \pm}$ is given by

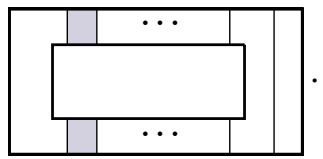

(2) If $x \in P_{n,-}$, then

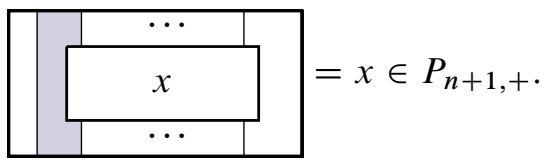

Proposition 2.47 (Conditional expectations). (1) The conditional expectation $\theta_{n-1}^{-1}$ 。 $E_{M_{n-1}} \circ \theta_{n}: P_{n,+} \rightarrow P_{n-1,+}$ is given by

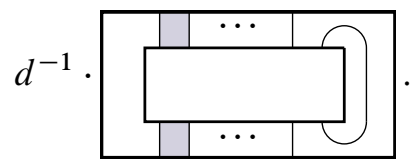


(2) The conditional expectation $\theta_{n}^{-1} \circ E_{M_{1}^{\prime}}^{M_{0}^{\prime}} \circ \theta_{n}: P_{n,+} \rightarrow P_{n-1,-}$ (see Proposition 2.24) is given by

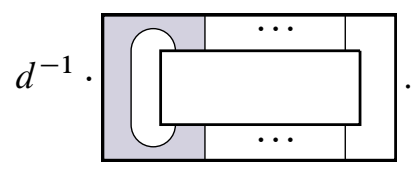

Notation 2.48. We use the notation from [16]. (1) Denote the annular capping maps $P_{n,+} \rightarrow P_{n-1,+}$ by $\alpha_{j}$ as shown:
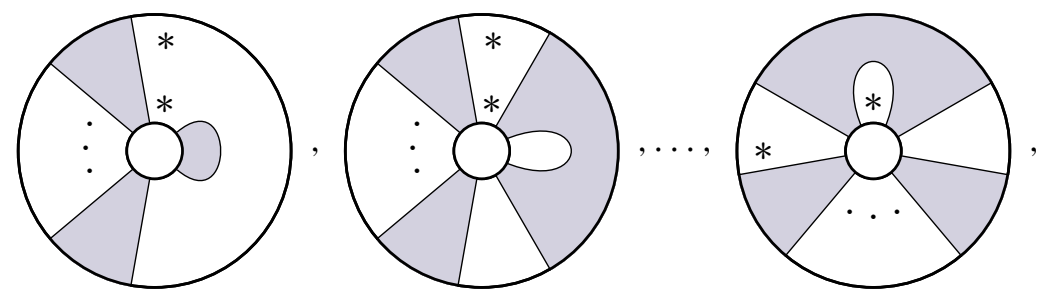

i.e. numbering the boundary points clockwise from $*$, the $i^{\text {th }}$ and $(i+1)^{\text {th }}$ (modulo $2 n$ ) internal boundary points are joined by a string and all other internal boundary points are connected to external boundary points such that

(i) if $i=1$, then the first external point is connected to the third internal point;

(ii) if $1<i<2 n$, then the first external point is connected to the first internal point;

(iii) if $i=2 n$, then the first external point is connected to the $(2 n-1)^{\text {th }}$ internal point.

(2) Denote the annular cupping maps $P_{n-1,+} \rightarrow P_{n,+}$ by $\beta_{j}$ as shown:
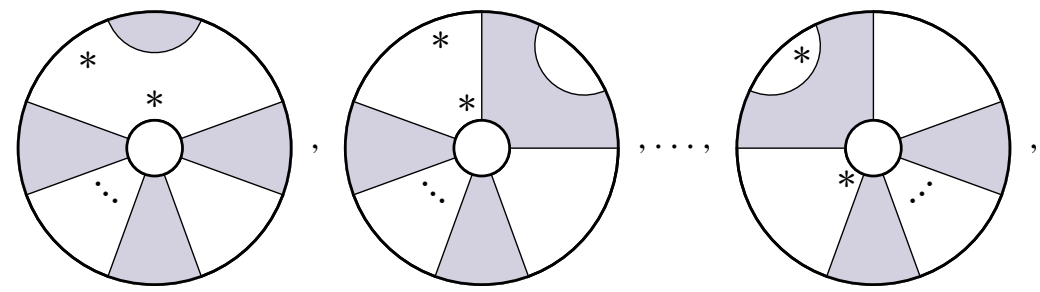

i.e. $\beta_{j}$ is $\alpha_{j}$ turned inside out.

The following lemma is similar to a result in [13].

Lemma 2.49. Suppose $P_{\bullet}$ is a planar $*$-algebra with modulus $d \neq 0$ and $Q_{n, \pm} \subset$ $P_{n, \pm}$ are $*$-subalgebras which are closed under the following operations:

(1) left and right multiplication by

$$
E_{n}=\begin{array}{|l|c|c|}
\hline{ }_{n-1} & \bigcirc
\end{array} \in P_{n+1,+}
$$


for $n \in \mathbb{N}$;

(2) the maps from $P_{n,+}$ as follows:

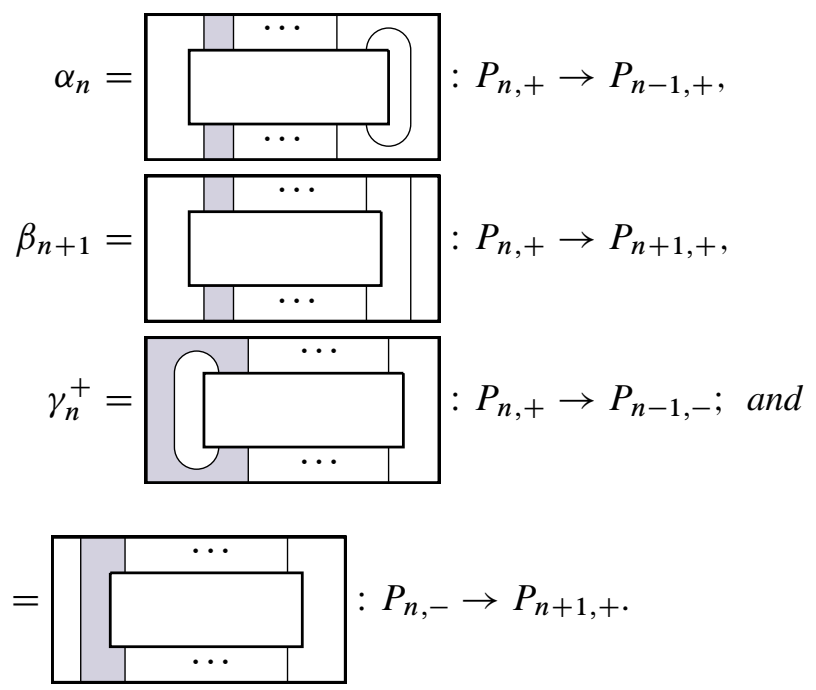

Then the $Q_{n, \pm}$ define a planar $*$-subalgebra $Q \bullet \subset P$ •.

Proof. As $Q_{n, \pm}$ is closed under multiplication and $*$, it suffices to show $Q \bullet$ is closed under all annular maps. To show this, it suffices to show all $\alpha_{j}$ 's, all $\beta_{j}$ 's, and both rotations by 1 preserve $Q$.

First, note that the maps $\gamma_{n}^{-}: P_{n,-} \rightarrow P_{n-1,+}$ and $i_{n}^{+}: P_{n,+} \rightarrow P_{n+1,-}$ by

$$
\begin{aligned}
& \gamma_{n}^{-}(x)=\begin{array}{|l|l|}
\hline & \cdots \\
\hline & x \\
\hline
\end{array} \\
& \left.=\frac{1}{d} \alpha_{n+2}\left(E_{n} E_{n-1} \ldots E_{1} \cdot \beta_{n+2}\left(i_{n}^{-} x\right)\right) \cdot E_{1} E_{2} \ldots E_{n}\right) \text {, } \\
& i_{n}^{+}(x)=\begin{array}{|l|}
\hline x \\
\hline x \\
\hline x
\end{array} \\
& =\gamma_{n+2}^{+}\left(E_{1} E_{2} \ldots E_{n} \cdot \beta_{n+2} \beta_{n+1}(x) \cdot E_{n+1} E_{n} \ldots E_{1}\right)
\end{aligned}
$$

preserve $Q$ •.

We show all $\alpha_{j}$ 's preserve $Q$. For $j<n$ and $x \in Q_{n}$,

$$
\left.\alpha_{j}(x)=\frac{1}{d} \alpha_{n} \alpha_{n+1}\left(\left(E_{n} E_{n-1} \ldots E_{j}\right) \cdot \beta_{n+1}(x) \cdot\left(E_{n}\right)\right)\right) .
$$

The case $n<j<2 n$ is similar. It is clear $\alpha_{2 n}(x)=\alpha_{2 n-1}\left(i_{n-1}^{-}\left(\gamma_{n}^{+}(x)\right)\right)$. 
We show all $\beta_{j}$ 's preserve $Q$. If $j<n+1$, we have

$$
\beta_{j}(x)=\left(E_{j} E_{j-1} \ldots E_{n}\right) \cdot \beta_{n+1}(x) .
$$

The case $n+1<j<2 n+2$ is similar. It is clear $\beta_{2 n+2}(x)=\alpha_{2} \gamma_{n+1}^{-} \gamma_{n}^{+}(x)$.

We show both rotations by 1 preserve $Q$. We have

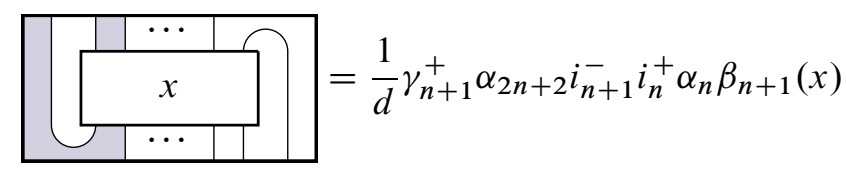

and

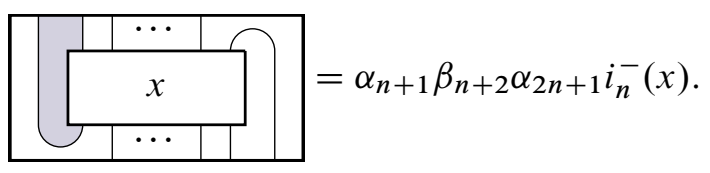

Theorem 2.50. Given a strongly Markov inclusion $M_{0} \subset\left(M_{1}, \operatorname{tr}_{1}\right)$, there is a unique planar $*$-algebra $P \bullet$ of modulus $d=\left[M_{1}: M_{0}\right]^{1 / 2}$ where

$$
P_{n,+}=\theta_{n}^{-1}\left(M_{0}^{\prime} \cap M_{n}\right) \text { and } P_{n,-}=\theta_{n+1}^{-1}\left(M_{1}^{\prime} \cap M_{n+1}\right)
$$

such that the multiplication is given by Remark 2.42,

(0) for all tangles $T$ with $n$ input disks, $T\left(\xi_{1}^{*}, \ldots, \xi_{n}^{*}\right)=T^{*}\left(\xi_{1}, \ldots, \xi_{n}\right)^{*}$ where, for $\xi_{i} \in P_{n_{i}, \pm_{i}}, \xi_{i}^{*}$ is as in Proposition 2.43 and $T^{*}$ is the mirror image of $T$;

(1) for $n \in \mathbb{N}$,

$$
E_{n}=\begin{array}{|l|l|}
\underbrace{}_{n-1} & \bigcirc
\end{array} \in P_{n+1,+} ;
$$

(2) for $x \in P_{n,+}$ and B a Pimsner-Popa basis for $M_{1}$ over $M_{0}$,

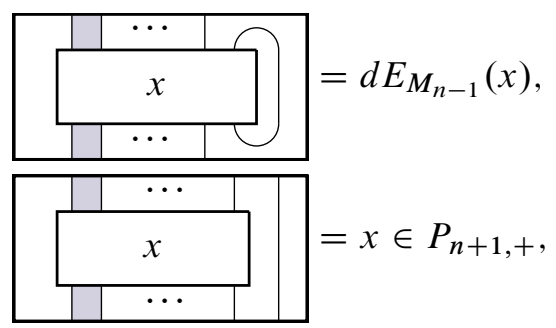

and

$$
\begin{array}{|l|l|}
\hline \begin{array}{c|c|}
\hline & \cdots \\
\hline & \cdots
\end{array} \\
\hline
\end{array}=d E_{M_{1}^{\prime}}^{M_{0}^{\prime}}(x)=d^{-1} \sum_{b \in B} b x b^{*} ;
$$

and 
(3) for $x \in P_{n,-}$,

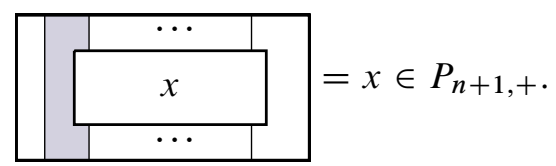

Proof. Uniqueness follows from Lemma 2.49. Existence follows from the existence of the canonical planar $*$-algebra associated to $M_{0} \subset\left(M_{1}, \operatorname{tr}_{1}\right)$.

Corollary 2.51. The canonical planar *-algebra associated to an extremal, finite index $I I_{1}$-subfactor is the subfactor planar algebra constructed in [9].

\section{The planar algebra isomorphism for finite dimensional $\mathrm{C}^{*}$-algebras}

We now restrict our attention to a connected unital inclusion $M_{0} \subset M_{1}$ of finite dimensional $\mathrm{C}^{*}$-algebras with the Markov trace. We show that in this case, the canonical planar $*$-algebra of Theorem 2.50 is isomorphic to the bipartite graph planar algebra [10] of the Bratteli diagram.

Many of the results in this section can be found in [6], [12], and [5], but we present them here for completeness and for the reader's convenience.

3.1. Loop algebras. We define loop algebras in the spirit of [10] which are another description of Evans, Ocneanu, and Sunder's path algebras [6], [12], and [5], with a more GNS (rather than spatial) flavor.

Notation 3.1. For this section, let $\Gamma$ be a finite, connected, bipartite multi-graph. Let $\mathcal{V}_{ \pm}$denote the set of even/odd vertices of $\Gamma$, and let $\mathcal{E}$ denote the edge set of $\Gamma$. Usually we will denote edges by $\varepsilon$ and $\xi$. All edges will be directed from even to odd vertices, so we have source and target functions $s: \mathcal{E} \rightarrow \mathcal{V}_{+}$and $t: \mathcal{E} \rightarrow \mathcal{V}_{-}$. We will write $\varepsilon^{*}$ to denote an edge $\varepsilon$ traversed from an odd vertex to an even vertex, and we define source and target functions $s: \mathcal{E}^{*}=\left\{\varepsilon^{*} \mid \varepsilon \in \mathcal{E}\right\} \rightarrow \mathcal{V}_{-}$and $t: \mathcal{E}^{*} \rightarrow \mathcal{V}_{+}$ by $s\left(\varepsilon^{*}\right)=t(\varepsilon)$ and $t\left(\varepsilon^{*}\right)=s(\varepsilon)$. Let $m_{+}: \mathcal{V}_{+} \rightarrow \mathbb{N}$ be a dimension (row) vector for the even vertices. For $v \in \mathcal{V}_{-}$, define the dimension (row) vector for the odd vertices by

$$
m_{-}(v)=\sum_{t(\varepsilon)=v} m_{+}(s(\varepsilon)) .
$$

Let $\Lambda$ be the bipartite adjacency matrix for $\Gamma\left(\Lambda_{i, j}\right.$ is the number of times the $i^{\text {th }}$ vertex in $\mathcal{V}_{+}$is connected to the $j^{\text {th }}$ vertex in $\mathcal{V}_{-}$).

Remark 3.2. Given $\left(\Gamma, m_{+}\right)$, we can associate a connected unital inclusion of finite dimensional $\mathrm{C}^{*}$-algebras $M_{0} \subset M_{1}$. We set

$$
M_{0}=\bigoplus_{v \in \mathcal{V}_{+}} M_{m_{+}(v)}(\mathbb{C}) \quad \text { and } \quad M_{1}=\bigoplus_{v \in \mathcal{V}_{-}} M_{m_{-}}(\mathbb{C}),
$$


and the inclusion is such that $\Gamma$ is the Bratteli diagram for the inclusion, and $\Lambda$ is the inclusion matrix $\left(\Lambda_{i, j}\right.$ is the number of times the $i^{\text {th }}$ simple summand of $M_{0}$ is contained in the $j^{\text {th }}$ simple summand of $M_{1}$ ). Conversely, given such an inclusion, we get a finite, connected, bipartite multi-graph (the Bratteli diagram) and a dimension vector $m_{+}$(corresponding to the simple summands of $M_{0}$ ).

Definition 3.3. Let $G_{0, \pm}$ be the complex vector space with basis $\mathcal{V}_{ \pm}$respectively. For $n \in \mathbb{N}, G_{n, \pm}$ will denote the complex vector space with basis loops of length $2 n$ on $\Gamma$ based at a vertex in $\mathcal{V}_{ \pm}$respectively.

We discuss the vector spaces $G_{n,+}$. The spaces $G_{n,-}$ are similar, and it is clear what the corresponding notation should be and how they will behave.

Notation 3.4. Loops in $G_{n,+}$ will be denoted $\left[\varepsilon_{1} \varepsilon_{2}^{*} \ldots \varepsilon_{2 n-1} \varepsilon_{2 n}^{*}\right]$. Any time we write such a loop, it is implied that

(i) $t\left(\varepsilon_{i}\right)=s\left(\varepsilon_{i+1}^{*}\right)=t\left(\varepsilon_{i+1}\right)$ for all odd $i<2 n$,

(ii) $t\left(\varepsilon_{i}^{*}\right)=s\left(\varepsilon_{i}\right)=s\left(\varepsilon_{i+1}\right)$ for all even $i<2 n$, and

(iii) $t\left(\varepsilon_{2 n}^{*}\right)=s\left(\varepsilon_{2 n}\right)=s\left(\varepsilon_{1}\right)$.

For a loop $\ell=\left[\varepsilon_{1} \varepsilon_{2}^{*} \ldots \varepsilon_{2 n-1} \varepsilon_{2 n}^{*}\right] \in G_{n,+}$ and $1 \leq k \leq 2 n$, we define the following paths in $\ell$ :

$\ell_{[1, k]}=\left\{\begin{array}{ll}\varepsilon_{1} \varepsilon_{2}^{*} \ldots \varepsilon_{k-1} \varepsilon_{k}^{*} & k \text { even, } \\ \varepsilon_{1} \varepsilon_{2}^{*} \ldots \varepsilon_{k-1}^{*} \varepsilon_{k} & k \text { odd, }\end{array} \quad \ell_{[k, 2 n]}= \begin{cases}\varepsilon_{k} \varepsilon_{k+1}^{*} \ldots \varepsilon_{2 n-1} \varepsilon_{2 n}^{*} & k \text { odd }, \\ \varepsilon_{k}^{*} \varepsilon_{k+1} \ldots \varepsilon_{2 n-1} \varepsilon_{2 n}^{*} & k \text { even. }\end{cases}\right.$

Definition 3.5. Define an antilinear map $*$ on $G_{n,+}$ by the antilinear extension of the map

$$
\left[\varepsilon_{1} \varepsilon_{2}^{*} \ldots \varepsilon_{2 n-1} \varepsilon_{2 n}^{*}\right]^{*}=\left[\varepsilon_{2 n} \varepsilon_{2 n-1}^{*} \ldots \varepsilon_{2} \varepsilon_{1}^{*}\right] .
$$

There is also an obvious notion of taking $*$ of a path in a loop. We define a multiplication on $G_{n,+}$ by

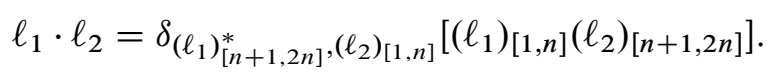

It is clear that $*$ is an involution, i.e. an anti-automorphism of period 2 , for $G_{n,+}$ under this multiplication.

Remark 3.6. We can think of a loop in $G_{n,+}$ as a path up and down the multi-graph $\Gamma_{n}$ corresponding to the Bratteli diagram for the inclusions

$$
M_{0} \subset M_{1} \subset \cdots \subset M_{n},
$$

which is obtained by reflecting $\Gamma$ a total of $n-1$ times, as the inclusion matrix of $M_{j} \subset M_{j+1}$ is given by $\Lambda$ or $\Lambda^{T}$ if $j$ is even or odd, respectively [8]. 
Definition 3.7. Let $\widetilde{\Gamma}$ be the augmentation of the bipartite graph $\Gamma$ by adding a distinguished vertex $\star$ which is connected to each $v \in \mathcal{V}_{+}$by $m_{+}(v)$ distinct edges. These edges are oriented so they begin at $\star$. We will denote these added edges by $\eta^{\prime} s$ (and ' 's and $\kappa$ 's when necessary).

Definition 3.8. For $n \in \mathbb{Z}_{\geq 0}$, let $A_{n}$ be the $\mathbb{C}$-algebra defined as follows: a basis of $A_{n}$ will consist of loops of length $2 n+2$ on $\widetilde{\Gamma}$ of the form

$$
\left[\eta_{1} \varepsilon_{1} \varepsilon_{2}^{*} \ldots \varepsilon_{2 n-1} \varepsilon_{2 n}^{*} \eta_{2}^{*}\right]
$$

i.e. the loops start and end at $\star$, but remain in $\Gamma$ otherwise. Note that we have an obvious $*$-structure on each $A_{n}$. Multiplication will be given as follows: if one defines the similar path notation as in Notation 3.4, then we have

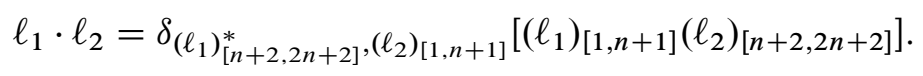

Remark 3.9. We can think of a loop in $A_{n}$ as a path up and down the multi-graph $\widetilde{\Gamma}_{n}$ corresponding to the Bratteli diagram for the inclusions

$$
\mathbb{C} \subset M_{0} \subset M_{1} \subset \cdots \subset M_{n} .
$$

Definition 3.10 (Inclusions). The inclusion $A_{n} \rightarrow A_{n+1}$ is given by the linear extension of

$$
\begin{aligned}
& {\left[\eta_{1} \varepsilon_{1} \varepsilon_{2}^{*} \ldots \varepsilon_{2 n-1} \varepsilon_{2 n}^{*} \eta_{2}^{*}\right]} \\
& \longmapsto \begin{cases}\sum_{s(\varepsilon)=s\left(\varepsilon_{n}\right)}\left[\eta_{1} \varepsilon_{1} \varepsilon_{2}^{*} \ldots \varepsilon_{n}^{*} \varepsilon \varepsilon^{*} \varepsilon_{n+1} \ldots \varepsilon_{2 n-1} \varepsilon_{2 n}^{*} \eta_{2}^{*}\right] & n \text { even, } \\
\sum_{s(\varepsilon)=t\left(\varepsilon_{n}\right)}\left[\eta_{1} \varepsilon_{1} \varepsilon_{2}^{*} \ldots \varepsilon_{n} \varepsilon^{*} \varepsilon \varepsilon_{n+1}^{*} \ldots \varepsilon_{2 n-1} \varepsilon_{2 n}^{*} \eta_{2}^{*}\right] & n \text { odd. }\end{cases}
\end{aligned}
$$

We identify $A_{n}$ with its image in $A_{n+1}$.

Remark 3.11. The inclusion identifications allow us to define a multiplication

$$
A_{m} \times A_{n} \rightarrow A_{\max \{m, n\}}
$$

by including $A_{m}, A_{n}$ into $A_{\max \{m, n\}}$ and using the regular multiplication. Explicitly, if $\ell_{1} \in A_{m}$ and $\ell_{2} \in A_{n}$ with $m \leq n$, then

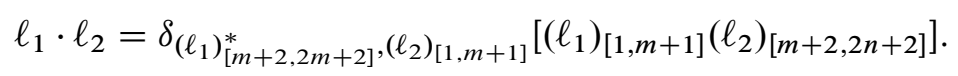

The case $m \geq n$ is similar. 
3.2. Towers of loop algebras. We provide an isomorphism of the tower $\left(M_{n}\right)_{n \geq 0}$ coming from a connected unital inclusion of finite dimensional $\mathrm{C}^{*}$-algebras with the Markov trace and the corresponding tower $\left(A_{n}\right)_{n \geq 0}$ of loop algebras. Assume the notation of Subsection 3.1.

For $n \geq 0$, if $S_{i}$ is the $i^{\text {th }}$ simple summand of $M_{n}$, then loops $\ell$ in $A_{n}$ for which $\ell_{[1, n+1]}$ ends at the corresponding vertex of $\widetilde{\Gamma}_{n}$ form a system of matrix units for a simple algebra isomorphic to $S_{i}$. Hence for $n \in \mathbb{Z}_{\geq 0}$, there is a $*$-algebra isomorphism $A_{n} \cong M_{n}$, and $\operatorname{dim}\left(A_{n}\right)=\operatorname{dim}\left(M_{n}\right)$.

At this point, we only choose such isomorphisms $\varphi_{n}: A_{n} \rightarrow M_{n}$ for $n=0,1$ which respect the inclusion given in Definition 3.10. In Proposition 3.17, we will inductively define isomorphisms $\varphi_{n}: A_{n} \rightarrow M_{n}$ for $n \geq 2$ to identify the Jones projections.

Definition 3.12. Following [8], let $\lambda_{i}$ be the Markov trace (column) vector for $M_{i}$ for $i=0,1$ such that

$$
m_{+} \lambda_{0}=1=m_{-} \lambda_{1},
$$

so $\lambda_{i}$ gives the traces of minimal projections in the simple summands of $M_{i}$ for $i=0,1$. In order for the trace on $M_{1}$ to restrict to the trace on $M_{0}$, we must have $\Lambda \lambda_{1}=\lambda_{0}$.

Recall that the inclusion matrix for $M_{n} \subset M_{n+1}$ is given by $\Lambda$ if $n$ is even and $\Lambda^{T}$ if $n$ is odd. This means that to extend the trace, we must have $\Lambda \Lambda^{T} \lambda_{0}=d^{-2} \lambda_{0}$, $\Lambda^{T} \Lambda \lambda_{1}=d^{-2} \lambda_{1}$, and $\lambda_{n}=d^{-2} \lambda_{n-2}$ for all $n \geq 2$, where $\lambda_{n}$ is the Markov trace vector for $M_{n}$ and $d=\sqrt{\left\|\Lambda^{T} \Lambda\right\|}=\sqrt{\left\|\Lambda \Lambda^{T}\right\|}$.

Definition 3.13. Let $\lambda=\left(\begin{array}{c}\lambda_{0} \\ d \lambda_{1}\end{array}\right)$, a Frobenius-Perron eigenvector for $\left(\begin{array}{cc}0 & \Lambda \\ \Lambda^{T} & 0\end{array}\right)$.

Definition 3.14 (Traces). We define a trace on $A_{0}$ by

$$
\operatorname{tr}_{0}\left(\left[\eta_{1} \eta_{2}^{*}\right]\right)= \begin{cases}\lambda\left(t\left(\eta_{1}\right)\right)=\lambda_{0}\left(t\left(\eta_{1}\right)\right) & \text { if } \eta_{1}=\eta_{2}, \\ 0 & \text { otherwise. }\end{cases}
$$

Suppose $\ell=\left[\eta_{1} \varepsilon_{1} \varepsilon_{2}^{*} \ldots \varepsilon_{2 n-1} \varepsilon_{2 n}^{*} \eta_{2}^{*}\right] \in A_{n}$ with $n \geq 1$. We define a trace on $A_{n}$ by

$$
\operatorname{tr}_{n}(\ell)= \begin{cases}d^{-n} \lambda\left(s\left(\varepsilon_{n}\right)\right) & \text { if } n \text { is even and } \ell=\ell^{*}, \\ d^{-n} \lambda\left(t\left(\varepsilon_{n}\right)\right) & \text { if } n \text { is odd and } \ell=\ell^{*}, \\ 0 & \text { if } \ell \neq \ell^{*}\end{cases}
$$

Remark 3.15. The isomorphisms $\varphi_{n}$ for $n=0,1$ preserve the trace. Moreover, $\left.\operatorname{tr}_{n+1}\right|_{A_{n}}=\operatorname{tr}_{n}$ for all $n \in \mathbb{N}$ as $\lambda$ is a Frobenius-Perron eigenvector. 
Proposition 3.16 (Conditional expectations). If $\ell=\left[\eta_{1} \varepsilon_{1} \varepsilon_{2}^{*} \ldots \varepsilon_{2 n-1} \varepsilon_{2 n}^{*} \eta_{2}^{*}\right] \in A_{n}$, the conditional expectation $A_{n} \rightarrow A_{n-1}$ is given by

$$
\begin{aligned}
& E_{A_{n-1}}(\ell) \\
& \quad=\left\{\begin{array}{l}
d^{-1} \delta_{\varepsilon_{n}, \varepsilon_{n+1}}\left(\frac{\lambda\left(s\left(\varepsilon_{n}\right)\right)}{\lambda\left(t\left(\varepsilon_{n}\right)\right)}\right)\left[\eta_{1} \varepsilon_{1} \varepsilon_{2}^{*} \ldots \varepsilon_{n-1} \varepsilon_{n+2}^{*} \ldots \varepsilon_{2 n-1} \varepsilon_{2 n}^{*} \eta_{2}^{*}\right] \quad n \text { even }, \\
d^{-1} \delta_{\varepsilon_{n}, \varepsilon_{n+1}}\left(\frac{\lambda\left(t\left(\varepsilon_{n}\right)\right)}{\lambda\left(s\left(\varepsilon_{n}\right)\right)}\right)\left[\eta_{1} \varepsilon_{1} \varepsilon_{2}^{*} \ldots \varepsilon_{n-1}^{*} \varepsilon_{n+2} \ldots \varepsilon_{2 n-1} \varepsilon_{2 n}^{*} \eta_{2}^{*}\right] \quad n \text { odd } .
\end{array}\right.
\end{aligned}
$$

Proof. We consider the case $n$ even. The case $n$ odd is similar. We must show $\operatorname{tr}_{n}(x y)=\operatorname{tr}_{n-1}\left(E_{A_{n-1}}(x) y\right)$ for all $x \in A_{n}$ and $y \in A_{n-1}$. It suffices to check when $x, y$ are loops. If

$$
x=\left[\eta_{1} \varepsilon_{1} \varepsilon_{2}^{*} \ldots \varepsilon_{2 n-1} \varepsilon_{2 n}^{*} \eta_{2}^{*}\right] \text { and } y=\left[\eta_{3} \xi_{1} \xi_{2}^{*} \ldots \xi_{2 n-3} \xi_{2 n-2}^{*} \eta_{4}^{*}\right],
$$

using the formula above, we have

$$
\begin{aligned}
\operatorname{tr}_{n-1}\left(E_{A_{n-1}}(x) y\right)= & d^{-1} \delta_{\varepsilon_{n}, \varepsilon_{n+1}} \delta_{x_{[n+2,2 n+2]}^{*}, y_{[1, n]}} \frac{\lambda\left(s\left(\varepsilon_{n}\right)\right)}{\lambda\left(t\left(\varepsilon_{n}\right)\right)} \\
& \operatorname{tr}_{n-1}\left(\left[\eta_{1} \varepsilon_{1} \varepsilon_{2}^{*} \ldots \varepsilon_{n-2}^{*} \varepsilon_{n-1} \xi_{n}^{*} \xi_{n+1} \ldots \xi_{2 n-3} \xi_{2 n-2}^{*} \eta_{4}^{*}\right]\right) \\
= & d^{-n} \delta_{x_{[n+2,2 n+2]}^{*}, y_{[1, n]}} \delta_{\varepsilon_{n}, \varepsilon_{n+1}} \delta_{y_{[n+1,2 n-2]}^{*}, x_{[1, n]}} \lambda\left(s\left(\varepsilon_{n}\right)\right) \\
= & \operatorname{tr}_{n}(x y) .
\end{aligned}
$$

Definition 3.17 (Jones projections). For $n \in \mathbb{N}$, define distinguished elements of $A_{n+1}$ as follows: if $n$ is odd, define

$$
\begin{aligned}
F_{n}=\sum_{\vec{\imath}} \sum_{t(\eta)=s\left(\varepsilon_{i_{1}}\right)} \frac{\left[\lambda\left(t\left(\varepsilon_{i_{n}}\right)\right) \lambda\left(t\left(\varepsilon_{i_{n+1}}\right)\right)\right]^{1 / 2}}{\lambda\left(s\left(\varepsilon_{i_{n}}\right)\right)} \\
{\left[\eta \varepsilon_{i_{1}} \varepsilon_{i_{2}}^{*} \ldots \varepsilon_{i_{n-1}}^{*} \varepsilon_{i_{n}} \varepsilon_{i_{n}}^{*} \varepsilon_{i_{n+1}} \varepsilon_{i_{n+1}}^{*} \varepsilon_{i_{n-1}} \ldots \varepsilon_{i_{2}} \varepsilon_{i_{1}}^{*} \eta^{*}\right] }
\end{aligned}
$$

where the sum is taken over all vectors $\vec{\imath}=\left(i_{1}, i_{2}, \ldots, i_{n+1}\right)$ such that

$$
\left[\varepsilon_{i_{1}} \varepsilon_{i_{2}}^{*} \ldots \varepsilon_{i_{n-1}}^{*} \varepsilon_{i_{n}} \varepsilon_{i_{n}}^{*} \varepsilon_{i_{n+1}} \varepsilon_{i_{n+1}}^{*} \varepsilon_{i_{n-1}} \ldots \varepsilon_{i_{2}} \varepsilon_{i_{1}}^{*}\right] \in G_{n+1,+} .
$$

If $n$ is even, then define

$$
\begin{aligned}
F_{n}=\sum_{\vec{i}} \sum_{t(\eta)=s\left(\varepsilon_{i_{1}}\right)} \frac{\left[\lambda\left(s\left(\varepsilon_{i_{n}}\right)\right) \lambda\left(s\left(\varepsilon_{i_{n+1}}\right)\right)\right]^{1 / 2}}{\lambda\left(t\left(\varepsilon_{i_{n}}\right)\right)} \\
{\left[\eta \varepsilon_{i_{1}} \varepsilon_{i_{2}}^{*} \ldots \varepsilon_{i_{n-1}} \varepsilon_{i_{n}}^{*} \varepsilon_{i_{n}} \varepsilon_{i_{n+1}}^{*} \varepsilon_{i_{n+1}} \varepsilon_{i_{n-1}}^{*} \ldots \varepsilon_{i_{2}} \varepsilon_{i_{1}}^{*} \eta^{*}\right] }
\end{aligned}
$$

with a similar limitation on the vectors $\vec{\imath}=\left(i_{1}, i_{2}, \ldots, i_{n+1}\right)$. 
Lemma 3.18. (1) $F_{n} x F_{n}=d E_{A_{n-1}}(x) F_{n}$, for all $x \in A_{n}$, and

(2) $\operatorname{tr}_{n+1}\left(x F_{n}\right)=d^{-1} \operatorname{tr}_{n}(x)$, for all $x \in A_{n}$, i.e. $E_{A_{n}}\left(F_{n}\right)=d^{-1}$.

Proof. We prove the case $n$ odd. The case $n$ even is similar.

(1) If $x=\left[\zeta_{1} \xi_{1} \xi_{2}^{*} \ldots \xi_{n-1} \xi_{n}^{*} \ldots \xi_{2 n-1} \xi_{2 n}^{*} \zeta_{2}^{*}\right] \in A_{n}$, then $F_{n} x F_{n}$ is given by

$$
\begin{aligned}
\sum_{\vec{\imath}} \sum_{t(\eta)=s\left(\varepsilon_{i_{1}}\right)} \frac{\left[\lambda\left(t\left(\varepsilon_{i_{n}}\right)\right) \lambda\left(t\left(\varepsilon_{i_{n+1}}\right)\right)\right]^{1 / 2}}{\lambda\left(s\left(\varepsilon_{i_{n}}\right)\right)} & {\left[\eta \varepsilon_{i_{1}} \ldots \varepsilon_{i_{n-1}}^{*} \varepsilon_{i_{n}} \varepsilon_{i_{n}}^{*} \varepsilon_{i_{n+1}} \varepsilon_{i_{n+1}}^{*} \varepsilon_{i_{n-1}} \ldots \varepsilon_{i_{1}}^{*} \eta^{*}\right] } \\
& x \sum_{\vec{\jmath} t(\kappa)=s\left(\varepsilon_{j_{1}}\right)} \sum \frac{\left[\lambda\left(t\left(\varepsilon_{j_{n}}\right)\right) \lambda\left(t\left(\varepsilon_{j_{n+1}}\right)\right)\right]^{1 / 2}}{\lambda\left(s\left(\varepsilon_{j_{n}}\right)\right)}\left[\kappa \varepsilon_{j_{1}} \ldots \varepsilon_{j_{n-1}}^{*} \varepsilon_{j_{n}} \varepsilon_{j_{n}}^{*} \varepsilon_{j_{n+1}} \varepsilon_{j_{n+1}}^{*} \varepsilon_{j_{n-1}} \ldots \varepsilon_{j_{1}}^{*} \kappa^{*}\right] \\
= & \sum_{s(\xi)=s\left(\xi_{n-1}\right)} \frac{\left[\lambda(t(\xi)) \lambda\left(t\left(\xi_{n+1}\right)\right)\right]^{1 / 2}}{\lambda(s(\xi))}\left[\zeta_{1} \xi_{1} \xi_{2}^{*} \ldots \xi_{n-1}^{*} \xi \xi^{*} \xi_{n} \xi_{n+1}^{*} \ldots \xi_{2 n-1} \xi_{2 n}^{*} \zeta_{2}^{*}\right] \\
& \sum_{\vec{\jmath} t(\kappa)=s\left(\varepsilon_{j_{1}}\right)} \frac{\left[\lambda\left(t\left(\varepsilon_{j_{n}}\right)\right) \lambda\left(t\left(\varepsilon_{j_{n+1}}\right)\right)\right]^{1 / 2}}{\lambda\left(s\left(\varepsilon_{j_{n}}\right)\right)}\left[\kappa \varepsilon_{j_{1}} \ldots \varepsilon_{j_{n-1}}^{*} \varepsilon_{j_{n}} \varepsilon_{j_{n}}^{*} \varepsilon_{j_{n+1}} \varepsilon_{j_{n+1}}^{*} \varepsilon_{j_{n-1}} \ldots \varepsilon_{j_{1}}^{*} \kappa^{*}\right] \\
= & \delta_{\xi_{n}, \xi_{n+1}} \frac{\lambda\left(t\left(\xi_{n}\right)\right)}{\lambda\left(s\left(\xi_{n}\right)\right)} \sum_{\substack{s(\xi)=s\left(\xi_{n-1}\right) \\
s(\varepsilon)=s\left(\xi_{n+2}\right)}} \frac{[\lambda(t(\varepsilon)) \lambda(t(\xi))]^{1 / 2}}{\lambda(s(\varepsilon))} \\
= & d E_{A_{n-1}}(x) F_{n} .
\end{aligned}
$$

(2) Another straightforward calculation.

Proposition 3.19 (Basic construction). For $n \in \mathbb{N}$, the inclusion $A_{n-1} \subset A_{n} \subset$ $\left(A_{n+1}, \operatorname{tr}_{n+1}, d^{-1} F_{n}\right)$ is standard. Hence for all $k \geq 0$, there are isomorphisms $\varphi_{k}: A_{k} \rightarrow M_{k}$ preserving the trace such that $\left.\varphi_{k+1}\right|_{A_{k}}=\varphi_{k}$ and $\varphi_{m}\left(F_{n}\right)=E_{n}$ for all $m>n$.

Proof. We construct the isomorphisms $\varphi_{n}$ for $n \geq 1$ by induction on $n$. The base case is finished. Suppose we have constructed $\varphi_{n}$ for $n \geq 1$. We know that $M_{n+1}=$ $M_{n} E_{n} M_{n}$ and $A_{n} \cong M_{n}$ via $\varphi_{n}$. By Lemmata 2.15 and 3.18, there is an algebra isomorphism $h_{n+1}: M_{n+1}=M_{n} E_{n} M_{n} \rightarrow A_{n} F_{n} A_{n} \subseteq A_{n+1}$ such that $E_{n} \mapsto F_{n}$. But $\operatorname{dim}\left(M_{n+1}\right)=\operatorname{dim}\left(A_{n+1}\right)$, so $A_{n+1}=A_{n} F_{n} A_{n}$, and we set $\varphi_{n+1}=h_{n+1}^{-1}$, which extends $\varphi_{n}$. Finally, note the $\varphi_{m}$ 's preserve the trace by construction and the uniqueness of the Markov trace.

3.3. Relative commutants are isomorphic to loop algebras. We provide isomorphisms between the relative commutants of the tower $\left(A_{n}\right)_{n \geq 0}$ and the spaces $G_{n, \pm}$. 
Proposition 3.20 (Central vectors). A basis for the central vectors $A_{0}^{\prime} \cap A_{n}$ is given by

$$
S_{0, n}=\left\{\sum_{t(\eta)=s\left(\varepsilon_{1}\right)}\left[\eta \varepsilon_{1} \varepsilon_{2}^{*} \ldots \varepsilon_{2 n-1} \varepsilon_{2 n}^{*} \eta^{*}\right] \in A_{n} \mid\left[\varepsilon_{1} \varepsilon_{2}^{*} \ldots \varepsilon_{2 n-1} \varepsilon_{2 n}^{*}\right] \in G_{n,+}\right\} .
$$

A basis for the central vectors $A_{1}^{\prime} \cap A_{n+1}$ is given by

$$
\begin{aligned}
& S_{1, n+1}=\left\{\sum_{t(\eta)=s(\varepsilon)}\left[\eta \varepsilon \varepsilon_{1}^{*} \varepsilon_{2} \ldots \varepsilon_{2 n-1}^{*} \varepsilon_{2 n} \varepsilon^{*} \eta^{*}\right]\right. \\
& t(\varepsilon)=t\left(\varepsilon_{1}\right) \\
& \left.\in A_{n+1} \mid\left[\varepsilon_{1}^{*} \varepsilon_{2} \ldots \varepsilon_{2 n-1}^{*} \varepsilon_{2 n}\right] \in G_{n,-}\right\} .
\end{aligned}
$$

Proof. Note that if $\left[\zeta_{1} \zeta_{2}^{*}\right] \in A_{0}$, then we have

$$
\begin{aligned}
& {\left[\zeta_{1} \zeta_{2}^{*}\right] \cdot \sum_{t(\eta)=s\left(\varepsilon_{1}\right)}\left[\eta \varepsilon_{1} \varepsilon_{2}^{*} \ldots \varepsilon_{2 n-1} \varepsilon_{2 n}^{*} \eta^{*}\right]} \\
& \quad=\sum_{t(\eta)=s\left(\varepsilon_{1}\right)} \delta_{\zeta_{2}, \eta}\left[\zeta_{1} \varepsilon_{1} \varepsilon_{2}^{*} \ldots \varepsilon_{2 n-1} \varepsilon_{2 n}^{*} \eta^{*}\right] \\
& =\left[\zeta_{1} \varepsilon_{1} \varepsilon_{2}^{*} \ldots \varepsilon_{2 n-1} \varepsilon_{2 n}^{*} \zeta_{2}^{*}\right] \\
& =\sum_{t(\eta)=s\left(\varepsilon_{1}\right)} \delta_{\eta, \zeta_{1}}\left[\eta \varepsilon_{1} \varepsilon_{2}^{*} \ldots \varepsilon_{2 n-1} \varepsilon_{2 n}^{*} \zeta_{2}^{*}\right] \\
& =\left(\sum_{t(\eta)=s\left(\varepsilon_{1}\right)}\left[\eta \varepsilon_{1} \varepsilon_{2}^{*} \ldots \varepsilon_{2 n-1} \varepsilon_{2 n}^{*} \eta^{*}\right]\right) \cdot\left[\zeta_{1} \zeta_{2}^{*}\right]
\end{aligned}
$$

Hence $S_{0, n} \subset A_{0}^{\prime} \cap A_{n}$. Similarly, $S_{1, n+1} \subset A_{1}^{\prime} \cap A_{n}$.

Suppose now that $x \in A_{0}^{\prime} \cap A_{n}$. Then since $1_{A_{0}}=\sum_{\eta}\left[\eta \eta^{*}\right]$, we have

$$
x=\left(\sum_{\eta}\left[\eta \eta^{*}\right]\right) x=\left(\sum_{\eta}\left[\eta \eta^{*}\right] \cdot\left[\eta \eta^{*}\right]\right) x=\sum_{\eta}\left[\eta \eta^{*}\right] \cdot x \cdot\left[\eta \eta^{*}\right] \in \operatorname{span}\left(S_{0, n}\right) .
$$

Similarly, $A_{1}^{\prime} \cap A_{n+1} \subseteq \operatorname{span}\left(S_{1, n+1}\right)$.

Corollary 3.21. There are $*$-algebra isomorphisms $\varphi_{n,+}: G_{n,+} \rightarrow A_{0}^{\prime} \cap A_{n}$ and $\varphi_{n,-}: G_{n,-} \rightarrow A_{1}^{\prime} \cap A_{n+1}$. If $n=0$, the isomorphisms are given by

$$
\varphi_{0,+}\left(v_{+}\right)=\sum_{t(\eta)=v_{+}}\left[\eta \eta^{*}\right] \text { and } \varphi_{0,-}\left(v_{-}\right)=\sum_{\substack{t(\eta)=s(\varepsilon) \\ t(\varepsilon)=v_{-}}}\left[\eta \varepsilon \varepsilon^{*} \eta^{*}\right] .
$$

For $n \in \mathbb{N}$, the isomorphisms are given by

$$
\varphi_{n,+}\left(\left[\varepsilon_{1} \varepsilon_{2}^{*} \ldots \varepsilon_{2 n-1} \varepsilon_{2 n}^{*}\right]\right)=\sum_{t(\eta)=s\left(\varepsilon_{1}\right)}\left[\eta \varepsilon_{1} \varepsilon_{2}^{*} \ldots \varepsilon_{2 n-1} \varepsilon_{2 n}^{*} \eta^{*}\right]
$$


and

$$
\varphi_{n,-}\left(\left[\varepsilon_{1}^{*} \varepsilon_{2} \ldots \varepsilon_{2 n-1}^{*} \varepsilon_{2 n}\right]\right)=\sum_{\substack{t(\eta)=s(\varepsilon) \\ t(\varepsilon)=t\left(\varepsilon_{1}\right)}}\left[\eta \varepsilon \varepsilon_{1}^{*} \varepsilon_{2} \ldots \varepsilon_{2 n-1}^{*} \varepsilon_{2 n} \varepsilon^{*} \eta^{*}\right] .
$$

It will be helpful to have an explicit Pimsner-Popa basis for $A_{1}$ over $A_{0}$.

Proposition 3.22 (Pimsner-Popa bases). For each $v_{+} \in \mathcal{V}_{+}$, pick a distinguished $\eta_{v_{+}}$with $t\left(\eta_{v_{+}}\right)=v_{+}$. Set

$$
B_{1}=\left\{\left(\frac{d \lambda\left(s\left(\varepsilon_{2}\right)\right)}{\lambda\left(t\left(\varepsilon_{2}\right)\right)}\right)^{1 / 2} \sum_{t(\eta)=s\left(\varepsilon_{1}\right)}\left[\eta \varepsilon_{1} \varepsilon_{2}^{*} \eta^{*}\right] \mid\left[\varepsilon_{1} \varepsilon_{2}^{*}\right] \in G_{1,+}\right\}
$$

and

$$
B_{2}=\left\{\left(\frac{d \lambda\left(s\left(\varepsilon_{2}\right)\right)}{\lambda\left(t\left(\varepsilon_{2}\right)\right)}\right)^{1 / 2}\left[\eta_{1} \varepsilon_{1} \varepsilon_{2}^{*} \eta_{s\left(\varepsilon_{2}\right)}^{*}\right] \mid s\left(\varepsilon_{1}\right) \neq s\left(\varepsilon_{2}\right)\right\} .
$$

Then $B=B_{1} \amalg B_{2}$ is a Pimsner-Popa basis for $A_{1}$ over $A_{0}$.

Proof. Suppose $x=\left[\zeta_{1} \xi_{1} \xi_{2}^{*} \zeta_{2}^{*}\right] \in A_{1}$. Case 1. Suppose that $s\left(\xi_{1}\right)=s\left(\xi_{2}\right)$, so $\left[\xi_{1} \xi_{2}^{*}\right] \in G_{1,+}$. If $b \in B_{2}$, then $E_{A_{0}}\left(b^{*} x\right)=0$ as the formula will have delta functions $\delta_{\xi_{i}, \varepsilon_{i}}$ for $i=1,2$. Hence we have

$$
\begin{aligned}
& \sum_{b \in B} b E_{A_{0}}\left(b^{*} x\right) \\
&=\sum_{b \in B_{1}} b E_{A_{0}}\left(b^{*} x\right) \\
&=\sum_{b \in B_{1}} \frac{d \lambda\left(s\left(\varepsilon_{2}\right)\right)}{\lambda\left(t\left(\varepsilon_{2}\right)\right)} \sum_{\substack{t(\eta)=s\left(\varepsilon_{1}\right) \\
t(\zeta)=s\left(\varepsilon_{1}\right)}}\left[\eta \varepsilon_{1} \varepsilon_{2}^{*} \eta^{*}\right] E_{A_{0}}\left(\left[\zeta \varepsilon_{2} \varepsilon_{1}^{*} \zeta^{*}\right] \cdot\left[\zeta_{1} \xi_{1} \xi_{2}^{*} \zeta_{2}^{*}\right]\right) \\
&=\sum_{b \in B_{1}} \frac{d \lambda\left(s\left(\varepsilon_{2}\right)\right)}{\lambda\left(t\left(\varepsilon_{2}\right)\right)} \sum_{t(\eta)=s\left(\varepsilon_{1}\right)} \delta_{\zeta_{1}, \zeta} \delta_{\xi_{1}, \varepsilon_{1}}\left[\eta \varepsilon_{1} \varepsilon_{2}^{*} \eta^{*}\right] E_{A_{0}}\left(\left[\zeta \varepsilon_{2} \xi_{2}^{*} \zeta_{2}^{*}\right]\right) \\
&=\sum_{b \in B_{1}} \frac{d \lambda\left(s\left(\varepsilon_{2}\right)\right)}{\lambda\left(t\left(\varepsilon_{2}\right)\right)} \sum_{t(\eta)=s\left(\xi_{1}\right)}\left[\eta \xi_{1} \varepsilon_{2}^{*} \eta^{*}\right] E_{A_{0}}\left(\left[\zeta_{1} \varepsilon_{2} \xi_{2}^{*} \zeta_{2}^{*}\right]\right) \\
&=\sum_{b \in B_{1}} \sum_{t(\eta)=s\left(\xi_{1}\right)} \delta_{\xi_{2}, \varepsilon_{2}}\left[\eta \xi_{1} \varepsilon_{2}^{*} \eta^{*}\right] \cdot\left[\zeta_{1} \zeta_{2}^{*}\right] \\
&= \\
&= x \\
&= x
\end{aligned}
$$

Case 2. Suppose that $s\left(\xi_{1}\right) \neq s\left(\xi_{2}\right)$. If $b \in B_{1}$, then, similarly, $E_{A_{0}}\left(b^{*} x\right)=0$. 
Hence

$$
\begin{aligned}
\sum_{b \in B} b E_{A_{0}}\left(b^{*} x\right) & =\sum_{b \in B_{2}} b E_{A_{0}}\left(b^{*} x\right) \\
& =\sum_{b \in B_{2}} \frac{d \lambda\left(s\left(\varepsilon_{2}\right)\right)}{\lambda\left(t\left(\varepsilon_{2}\right)\right)}\left[\eta_{1} \varepsilon_{1} \varepsilon_{2}^{*} \eta_{s\left(\varepsilon_{2}\right)}^{*} E_{A_{0}}\left(\left[\eta_{s}\left(\varepsilon_{2}\right) \varepsilon_{2} \varepsilon_{1}^{*} \eta_{1}^{*}\right] \cdot\left[\zeta_{1} \xi_{1} \xi_{2}^{*} \zeta_{2}^{*}\right]\right)\right. \\
& =\left[\zeta_{1} \xi_{1} \xi_{2}^{*} \eta_{s\left(\xi_{2}\right)}^{*}\right] \cdot\left[\eta_{s\left(\xi_{2}\right)} \zeta_{2}^{*}\right]=\left[\zeta_{1} \xi_{1} \xi_{2}^{*} \zeta_{2}\right]=x
\end{aligned}
$$

Remark 3.23. One could also take

$$
B_{2}=\left\{\left(\frac{d \lambda\left(s\left(\varepsilon_{2}\right)\right)}{m_{+}\left(s\left(\varepsilon_{2}\right)\right) \lambda\left(t\left(\varepsilon_{2}\right)\right)}\right)^{1 / 2}\left[\eta_{1} \varepsilon_{1} \varepsilon_{2}^{*} \eta_{2}^{*}\right] \mid s\left(\varepsilon_{1}\right) \neq s\left(\varepsilon_{2}\right)\right\} .
$$

Corollary 3.24 (Commutant conditional expectations). If

$$
x=\sum_{t(\zeta)=s\left(\xi_{1}\right)}\left[\zeta \xi_{1} \xi_{2}^{*} \ldots \xi_{2 n-1} \xi_{2 n}^{*} \zeta^{*}\right] \in A_{0}^{\prime} \cap A_{n}
$$

the conditional expectation $A_{0}^{\prime} \cap A_{n} \rightarrow A_{1}^{\prime} \cap A_{n}$ is given by

$$
E_{A_{1}^{\prime}}^{A_{0}^{\prime}}(x)=d^{-1} \delta_{\xi_{1}, \xi_{2 n}}\left(\frac{\lambda\left(s\left(\xi_{1}\right)\right)}{\lambda\left(t\left(\xi_{1}\right)\right)}\right) \sum_{\substack{t(\zeta)=s(\varepsilon) \\ t(\varepsilon)=t\left(\xi_{2}\right)}}\left[\eta \xi_{2}^{*} \xi_{3} \ldots \xi_{2 n-2}^{*} \xi_{2 n-1} \varepsilon^{*} \eta^{*}\right]
$$

Proof. Let $B$ be as in Proposition 3.22. By Proposition 2.24, we have

$$
d^{2} E_{A_{1}^{\prime}}^{A_{0}^{\prime}}(x)=\sum_{b \in B} b x b^{*}=\sum_{b \in B_{1}} b x b^{*}+\sum_{b \in B_{2}} b x b^{*} .
$$

We treat each sum separately. We have $\sum_{b \in B_{1}} b x b^{*}$ is given by

$$
\begin{aligned}
& \sum_{b \in B_{1}} \frac{d \lambda\left(s\left(\varepsilon_{2}\right)\right)}{\lambda\left(t\left(\varepsilon_{2}\right)\right)} \sum_{\substack{t(\eta)=s\left(\varepsilon_{1}\right)=t(\kappa) \\
t(\zeta)=s\left(\xi_{1}\right)}}\left[\eta \varepsilon_{1} \varepsilon_{2}^{*} \eta^{*}\right] \cdot\left[\zeta \xi_{1} \xi_{2}^{*} \ldots \xi_{2 n-1} \xi_{2 n}^{*} \zeta^{*}\right] \cdot\left[\kappa \varepsilon_{2} \varepsilon_{1}^{*} \kappa^{*}\right] \\
& =d \sum_{\substack{s(\varepsilon)=s\left(\varepsilon_{2}\right) \\
t(\varepsilon)=t\left(\xi_{2}\right)}} \frac{\lambda\left(s\left(\varepsilon_{2}\right)\right)}{\lambda\left(t\left(\varepsilon_{2}\right)\right)} \sum_{\substack{t(\eta)=s\left(\varepsilon_{1}\right)=t(\kappa) \\
t(\zeta)=s\left(\xi_{1}\right)}} \delta_{\eta, \zeta} \delta_{\zeta, \kappa} \delta_{\varepsilon_{2}, \xi_{1}} \delta_{\varepsilon_{2}, \xi_{2 n}}\left[\eta \varepsilon \xi_{2}^{*} \ldots \xi_{2 n-1} \varepsilon^{*} \kappa^{*}\right] \\
& =d \sum_{\substack{t(\eta)=s(\varepsilon)=s\left(\xi_{1}\right) \\
t(\varepsilon)=t\left(\xi_{2}\right)}} \frac{\lambda\left(s\left(\xi_{1}\right)\right)}{\lambda\left(t\left(\xi_{1}\right)\right)} \delta_{\xi_{1}, \xi_{2 n}}\left[\eta \varepsilon \xi_{2}^{*} \ldots \xi_{2 n-1} \varepsilon^{*} \eta^{*}\right] .
\end{aligned}
$$


Similarly, we have

$$
\sum_{b \in B_{2}} b x b^{*}=d \sum_{\substack{t(\eta)=s(\varepsilon) \neq s\left(\xi_{1}\right) \\ t(\varepsilon)=t\left(\xi_{2}\right)}} \frac{\lambda\left(s\left(\xi_{1}\right)\right)}{\lambda\left(t\left(\xi_{1}\right)\right)} \delta_{\xi_{1}, \xi_{2 n}}\left[\eta \varepsilon \xi_{2}^{*} \ldots \xi_{2 n-1} \varepsilon^{*} \eta^{*}\right] .
$$

Putting these two together, we get the desired formula for $E_{A_{1}^{\prime}}^{A_{0}^{\prime}}$.

3.4. The bipartite graph planar algebra and the isomorphism. We refer the reader to [10] for the full definition of the planar algebra of a bipartite graph.

Let $G_{\bullet}$ be the planar algebra of the bipartite graph $\Gamma$ with spin vector $\lambda$ as in Subsections 3.1 and 3.2. We briefly recall the action of tangles on the $G_{n, \pm}$ and we calculate some necessary examples.

A state $\sigma$ of a tangle $T$ is a way of assigning the regions and strings of $T$ with compatible vertices and edges of $\Gamma$ respectively, i.e. if a string $S$ of $T$ partitions the unshaded region $R_{+}$from the shaded region $R_{-}$, then for $\sigma(S) \in \mathcal{E}, s(\sigma(S))=$ $\sigma\left(R_{+}\right) \in \mathcal{V}_{+}$and $t(\sigma(S))=\sigma\left(R_{-}\right) \in \mathcal{V}_{-}$.

Define the output loop $\ell_{\sigma}$ as the loop obtained by reading clockwise around the outer boundary of $T$ once it has been labelled by $\sigma$.

Suppose now that $T$ has $n$ input disks, and $\ell=\ell_{1} \otimes \cdots \otimes \ell_{n}$ is a simple tensor of loops where $\ell_{i}$ is a loop in $G_{n_{i}, \pm_{i}}$. Then the action of $T$ on $\ell$ is given by

$$
T(\ell)=\sum_{\text {states } \sigma} c(\sigma, \ell) \ell_{\sigma},
$$

where $c(\sigma, \ell)$ is a correction factor defined as follows.

(1) First, label the regions and strings of $T$ adjacent to the input disks with the edges and vertices which compose the $\ell_{i}$ 's. If the labelling contradicts $\sigma$, then $c(\sigma, \ell)=0$.

(2) If the labels agree, put the tangle in a standard form similar to Section 2.3, where the only difference is that the half the strings emanate from the top of the input rectangles, and half the strings emanate down, but the $*$ is still on the left side. Let $E(T)$ be the set of local extrema of the strings of the standard form of the tangle. For each $e \in E(T)$, let conv $(e)$ be the vertex assigned by $\sigma$ to the convex region of the extrema, and let conc $(e)$ be the vertex assigned to the concave region. Set

$$
k_{e}=\sqrt{\frac{\lambda(\operatorname{conv}(e))}{\lambda(\operatorname{conc}(e))}} .
$$

Below is an example of an extrema $e$ on a string $S$ with $\sigma(S)=\varepsilon$, connecting vertices $w, v$ :

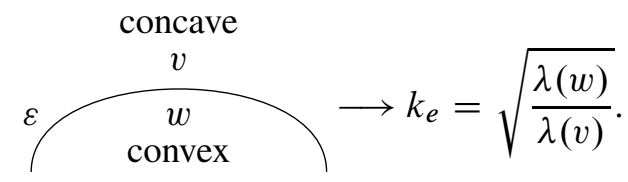


Note that $\operatorname{conv}(e)$ may be in either $\mathcal{V}_{+}$or $\mathcal{V}_{-}$. Finally, set

$$
c(\sigma, \ell)=\prod_{e \in E(T)} k_{e}
$$

The $*$-structure on the bipartite graph planar algebra is given as follows: if $T, \ell$ are as above, then

$$
T\left(\ell_{1}^{*} \otimes \cdots \otimes \ell_{n}^{*}\right)=T^{*}\left(\ell_{1} \otimes \cdots \otimes \ell_{n}\right)^{*},
$$

where $T^{*}$ is the mirror image of $T$, and the adjoint of a loop is the loop traversed backwards as in Definition 3.5.

Remark 3.25. Contractible loops are traded for a multiplicative factor of $d$ as $\lambda$ is a Frobenius-Perron eigenvector (see Definition 3.13).

Remark 3.26. Note from Corollary 3.21 that there is a natural inclusion identification $G_{n,-} \rightarrow G_{n+1,+}$ given by

$$
\left[\varepsilon_{1}^{*} \varepsilon_{2} \ldots \varepsilon_{2 n-1}^{*} \varepsilon_{2 n}\right] \longmapsto \sum_{t(\varepsilon)=s\left(\varepsilon_{1}\right)}\left[\varepsilon \varepsilon_{1}^{*} \varepsilon_{2} \ldots \varepsilon_{2 n-1}^{*} \varepsilon_{2 n} \varepsilon^{*}\right]
$$

Examples 3.27. (0) If $\ell_{1}, \ell_{2} \in G_{n, \pm}$, then

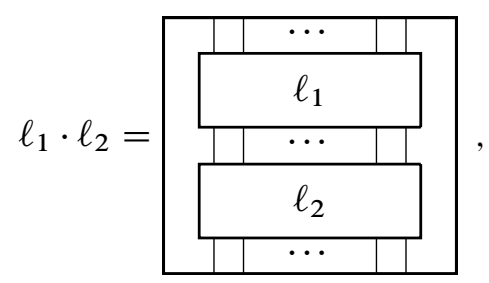

the shading depending on $n, \pm$.

(1) For $n \in \mathbb{N}$ odd,

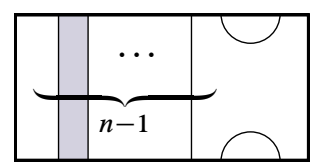

is equal to

$$
\sum_{\vec{l}} \frac{\left[\lambda\left(t\left(\varepsilon_{i_{n}}\right)\right) \lambda\left(t\left(\varepsilon_{i_{n+1}}\right)\right)\right]^{1 / 2}}{\lambda\left(s\left(\varepsilon_{i_{n}}\right)\right)}\left[\varepsilon_{i_{1}} \ldots \varepsilon_{i_{n-1}}^{*} \varepsilon_{i_{n}} \varepsilon_{i_{n}}^{*} \varepsilon_{i_{n+1}} \varepsilon_{i_{n+1}}^{*} \varepsilon_{i_{n-1}} \ldots \varepsilon_{i_{1}}^{*}\right],
$$

where the sum is taken over all vectors $\vec{\imath}=\left(i_{1}, i_{2}, \ldots, i_{n+1}\right)$ such that

$$
\left[\varepsilon_{i_{1}} \varepsilon_{i_{2}}^{*} \ldots \varepsilon_{i_{n-1}}^{*} \varepsilon_{i_{n}} \varepsilon_{i_{n}}^{*} \varepsilon_{i_{n+1}} \varepsilon_{i_{n+1}}^{*} \varepsilon_{i_{n-1}} \ldots \varepsilon_{i_{2}} \varepsilon_{i_{1}}^{*}\right] \in G_{n+1,+} .
$$

There is a similar formula for $n$ even. (Compare with Definition 3.17.)

(2) Suppose $\ell=\left[\varepsilon_{1} \varepsilon_{2}^{*} \ldots \varepsilon_{2 n-1} \varepsilon_{2 n}^{*}\right] \in G_{n,+}$. 
(i) If $n$ is even, then

$$
\begin{array}{|c|c|c|}
\hline & \cdots & \\
\hline & \ell \\
\hline & \cdots & \cdots
\end{array}=\delta_{\varepsilon_{n}, \varepsilon_{n+1}} \frac{\lambda\left(s\left(\varepsilon_{n}\right)\right)}{\lambda\left(t\left(\varepsilon_{n}\right)\right)}\left[\varepsilon_{1} \varepsilon_{2}^{*} \ldots \varepsilon_{n-1} \varepsilon_{n+2}^{*} \varepsilon_{2 n-1} \ldots \varepsilon_{2 n}^{*}\right],
$$

with a similar formula for $n$ odd. (Compare with Proposition 3.16.)

(ii) If $n$ is even, then

$$
\begin{array}{|l|l|}
\hline & \cdots \\
\hline & \ell \\
\hline & \cdots
\end{array}=\sum_{s(\varepsilon)=s\left(\varepsilon_{n}\right)}\left[\varepsilon_{1} \varepsilon_{2}^{*} \ldots \varepsilon_{n}^{*} \varepsilon \varepsilon^{*} \varepsilon_{n+1} \ldots \varepsilon_{2 n-1} \varepsilon_{2 n}^{*}\right],
$$

with a similar formula for $n$ odd. (Compare with Definition 3.10.)

(iii)

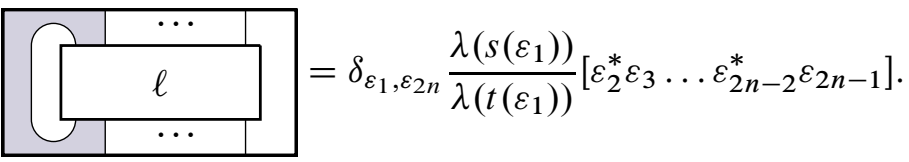

(Compare with Proposition 3.24 and Remark 3.26.)

(3) If $\ell=\left[\varepsilon_{1}^{*} \varepsilon_{2} \ldots \varepsilon_{2 n-1}^{*} \varepsilon_{2 n}\right] \in G_{n,-}$, then

$$
\begin{array}{|c|}
\frac{\ell}{\cdots} \\
\frac{\ell}{\cdots}
\end{array}=\sum_{t(\varepsilon)=s\left(\varepsilon_{1}\right)}\left[\varepsilon \varepsilon_{1}^{*} \varepsilon_{2} \ldots \varepsilon_{2 n-1}^{*} \varepsilon_{2 n} \varepsilon^{*}\right]
$$

which may be identified with $\ell \in G_{n+1,+}$ by Remark 3.26.

Theorem 3.28. The canonical planar $*$-algebra $P_{\bullet}$ associated to $M_{0} \subset\left(M_{1}, \operatorname{tr}_{1}\right)$ is isomorphic to the bipartite graph planar $*$-algebra $G \bullet$ of the Bratteli diagram $\Gamma$ for the inclusion.

Proof. To show that the $*$-algebra isomorphisms

$$
\begin{gathered}
G_{n,+} \stackrel{\varphi_{n,+}}{\longrightarrow} A_{0}^{\prime} \cap A_{n} \stackrel{\left.\varphi_{n}\right|_{A_{0}^{\prime} \cap A_{n}} ^{\longrightarrow}}{\longrightarrow} M_{0}^{\prime} \cap M_{n} \stackrel{\left.\theta_{n}^{-1}\right|_{M_{0}^{\prime} \cap M_{n}} ^{\longrightarrow}}{\longrightarrow} P_{n,+} \\
G_{n,-} \stackrel{\varphi_{n,-}}{\longrightarrow} A_{1}^{\prime} \cap A_{n+1} \stackrel{\left.\varphi_{n+1}\right|_{A_{1}^{\prime} \cap A_{n+1}} ^{\longrightarrow}}{\longrightarrow} M_{1}^{\prime} \cap M_{n+1} \stackrel{\left.\theta_{n+1}^{-1}\right|_{M_{1}^{\prime} \cap M_{n+1}} ^{\longrightarrow}}{\longrightarrow} P_{n,-}
\end{gathered}
$$

give an isomorphism of planar $*$-algebras $G_{\bullet} \rightarrow P_{\bullet}$, we must check that

(1) they map Jones projections in $G_{\bullet}$ to those in $P_{\bullet}$, and

(2) they preserve the action of annular tangles.

Both follow immediately from Examples 3.27 and the proof of Lemma 2.49. 


\section{The embedding theorem}

Let $Q$. be a finite depth subfactor planar algebra of modulus $d$. Pick $r \geq 0$ minimal such that $Q_{2 r,+} \subset Q_{2 r+1,+} \subset\left(Q_{2 r+2,+}, e_{2 r+1}\right)$ is standard (with the usual trace). Note this is possible if and only if $Q$. has finite depth. In fact, $Q_{k,+} \subset Q_{k+1,+} \subset\left(Q_{k+2,+}, e_{k+1}\right)$ is standard for all $k \geq 2 r$. For $n \geq 0$, set $M_{n}=Q_{2 r+n,+}$ and $F_{n+1}=E_{2 r+n+1}$ (shifted Jones projections). Let $P_{\bullet}$ be the canonical planar $*$-algebra associated to the inclusion $M_{0} \subset M_{1}$, i.e.

$$
P_{n,+}=M_{0}^{\prime} \cap M_{n}=Q_{2 r,+}^{\prime} \cap Q_{2 r+n,+}
$$

and

$$
P_{n,-}=M_{1}^{\prime} \cap M_{n+1}=Q_{2 r+1,+}^{\prime} \cap Q_{2 r+n+1,+},
$$

where we suppress the isomorphisms $\theta_{n}$ with the tensor products of $Q_{2 r+1,+}$ over $Q_{2 r,+}$.

Theorem 4.1. Define $\Phi: Q_{\bullet} \rightarrow P$. by adding $2 r$ strings to the left for $x \in Q_{n,+}$ and adding $2 r+1$ strings to the left for $x \in Q_{n,-}$ :

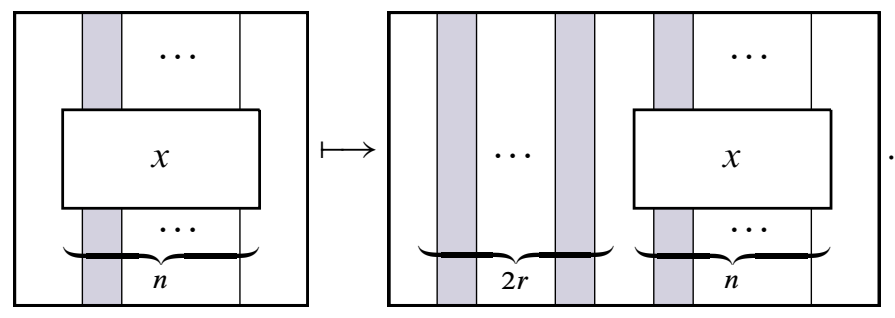

Then $\Phi$ is an inclusion of planar $*$-algebras.

Proof. We use Lemma 2.49. Note that $\Phi\left(x^{*}\right)=\Phi(x)^{*}$ and $\Phi(x y)=\Phi(x) \Phi(y)$ for all $x, y \in Q_{n, \pm}$.

(1) Since $\Phi\left(E_{j}\right)=E_{2 r+j}=F_{j}$ for all $j \in \mathbb{N}$, we have $\Phi\left(E_{j} x\right)=F_{j} \Phi(x)$ and $\Phi\left(x E_{j}\right)=\Phi(x) F_{j}$ for all $x \in Q_{n, \pm}$ and all $j \in \mathbb{N}$.

(2) Note that

(i) For $n \in \mathbb{N}, \Phi\left(E_{Q_{n-1,+}}(x)\right)=E_{P_{n-1,+}}(\Phi(x))$ since

$$
\left.E_{Q_{2 r+n-1,+}}\right|_{Q_{2 r,+}^{\prime} \cap Q_{2 r+n,+}}=\left.E_{Q_{2 r+n-1,+}}\right|_{P_{n,+}}=E_{P_{n-1,+}}
$$

(since $Q_{2 r,+} \subset Q_{2 r+n-1,+}$, we have that $E_{Q_{2 r+n-1,+}}$ preserves $Q_{2 r,+}$-central vectors as it is $Q_{2 r+n-1,+}$-bilinear).

(ii) $\Phi\left(\beta_{n+1}(x)\right)=\beta_{n+1}(\Phi(x))$ for all $x \in Q_{n,+}$ since the inclusion $P_{n,+} \rightarrow$ $P_{n+1,+}$ is the restriction of the inclusion $Q_{2 r+n,+} \rightarrow Q_{2 r+n+1,+}$. 
(iii) Let $B=\{b\}$ be a Pimsner-Popa basis for $M_{1}=Q_{2 r+1,+}$ over $M_{0}=Q_{2 r,+}$. Since each $b \in B$ is an $(2 r+1,+)$-box in $Q_{2 r+1,+}$,

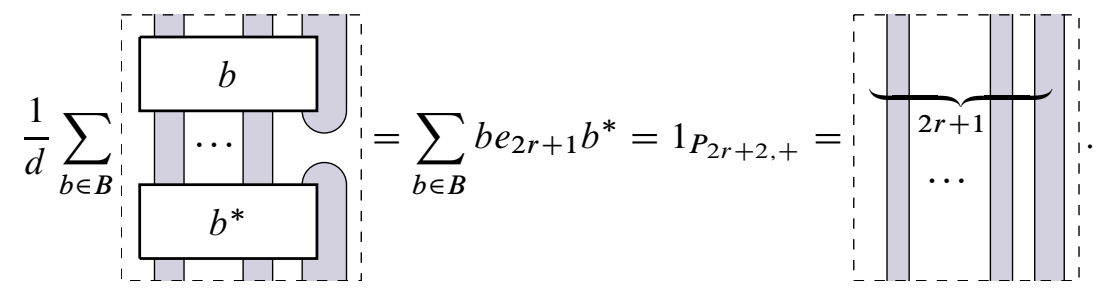

Then by Proposition 2.24 and Theorem 2.50, for all $x \in Q_{n,+}$,

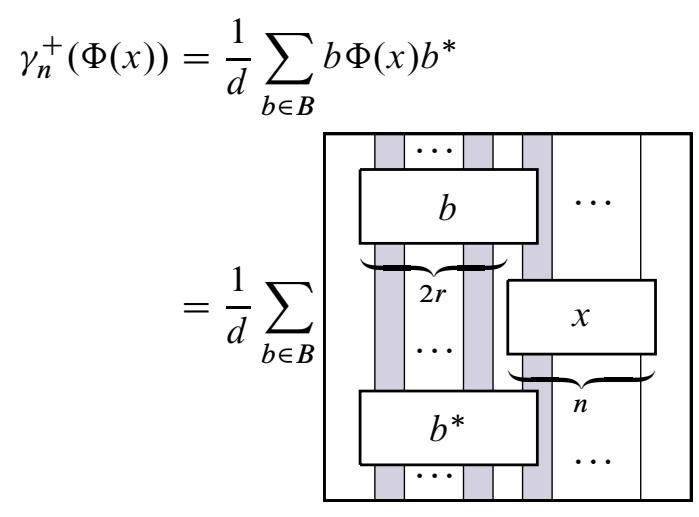

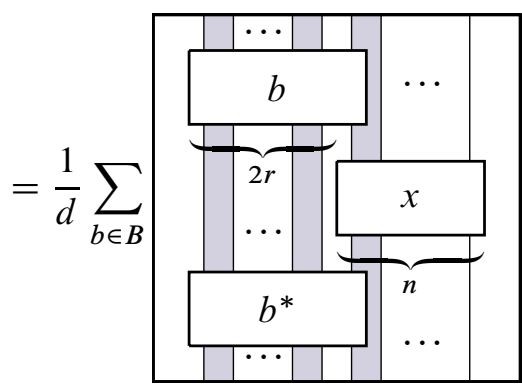

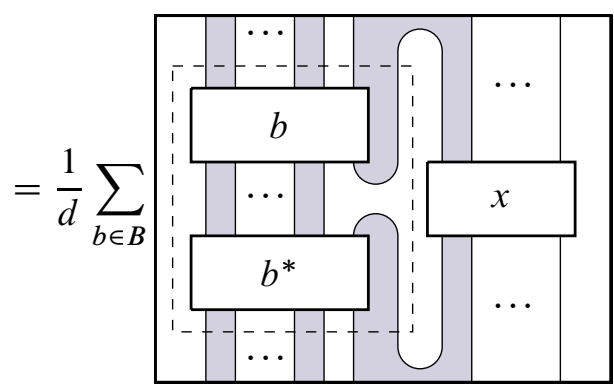

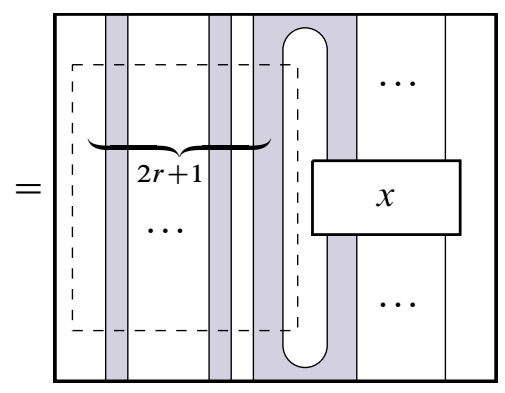

$$
=\Phi\left(\gamma_{n}^{+}(x)\right) \text {. }
$$


(3) The inclusion $i_{n}^{-}: P_{n,-} \rightarrow P_{n+1,+}$ is the identity in the canonical planar *-algebra. If $x \in Q_{n,-}$, then we have

$$
i_{n}^{-}(\Phi(x))=\Phi(x)=\begin{array}{|l|l|l|l|l|l|}
\hline & & \ldots & & & \cdots \\
\hline & & & & x \\
\hline & 2 r+1 & & \ldots & \\
\hline
\end{array}
$$

Corollary 4.2. Let $N \subset M$ be a finite index, finite depth $\mathrm{II}_{1}$-subfactor, and let $P$. be the associated canonical subfactor planar algebra. Let $\Gamma$ be the principal graph of $N \subset M$, and let $G_{\bullet}$ be the bipartite graph planar algebra of $\Gamma$. Then there is an embedding of planar algebras $P_{\bullet} \rightarrow G_{\bullet}$.

\section{References}

[1] M. Baillet, Y. Denizeau, and J.-F. Havet, Indice d'une espérance conditionelle. Compositio Math. 66 (1988), 199-236. MR 945550 Zbl 0657.46041

[2] S. Bigelow, S. Morrison, E. Peters, and N. Snyder, Constructing the extended Haagerup planar algebra. Preprit 2009. arXiv:0909.4099. To appear in Acta Math.

[3] D. Bisch, Bimodules, higher relative commutants and the fusion algebra associated to a subfactor. In Fillmore, Peter A. (ed.) et al., Operator algebras and their applications (Waterloo, ON, 1994/1995), 13-63. Amer. Math. Soc., Providence, RI, 1997. Google Books_InIRTO8Y7gC MR 1424954 Zbl 0894.46046

[4] M. Burns, Subfactors, planar algebras, and rotations. University of California Berkeley, 2003. Ph.D. Thesis.

[5] D. E. Evans and Y. Kawahigashi, Quantum Symmetries on Operator Algebras. Clarendon Press, Oxford, 1998. MR 1642584 Zbl 0924.46054

[6] F. M. Goodman, P. de la Harpe, and V. F. R. Jones, Coxeter graphs and towers of algebras. Springer Verlag, New York, 1989. MR 999799 Zbl 0698.46050

[7] P. Jolissaint, Index for pairs of finite von Neumann algebras. Pacific J. Math 146 (1990), 43-70. Euclid euclid.pjm/1102645309 MR 1073519 Zbl 0663.46052

[8] V. F. R. Jones, Index for subfactors. Invent. Math., 72 (1993), 1-25. MR 696688 Zbl 0508.46040

[9] V. F. R. Jones, Planar algebras I. Preprint 1999. arXiv:math/9909027.

[10] V. F. R. Jones, The planar algebra of a bipartite graph. In C. McA. Gordon (ed.) et al., Knots in Hellas '98 (Delphi), 94-117. World Sci. Publ., River Edge, NJ, 2000. http://math.berkeley.edu/ vfr/delphi.ps MR 1865703 Zbl 1021.46047

[11] V. F. R. Jones, Quadratic tangles in planar algebras. Prerpint 2010. arXiv:1007.1158v2

[12] V. F.R. Jones and V. S. Sunder, Introduction to subfactors. Cambridge University Press, Cambridge, 1997. MR 1473221 Zbl 0903.46062 
[13] V. Kodiyalam and V. S. Sunder, On Jones' planar algebras. J. Knot Theory Ramifications 13 (2004), 219-247. MR 2047470 Zbl 1054.46045

[14] S. Morrison, E. Peters, and N. Snyder, Skein theory for the $D_{2 n}$ planar algebras. J. Pure Appl. Alg. 214 (2010), 117-139. Zbl 1191.46051

[15] S. Morrison and K. Walker, The graph planar algebra embedding theorem, Preprint 2010. http://tqft.net/gpa

[16] D. Penneys, A cyclic approach to the annular Temperley-Lieb category, Preprint 2009. arXiv:0912.1320

[17] E. Peters, A planar algebra construction of the Haagerup subfactor. Internat. J. Math., 21 (2010), 987-1045. MR 2679382 Zbl 1203.46039

[18] M. Pimsner and S. Popa, Entropy and index for subfactors. Ann. Sci. École Norm. Sup. (4) 19 (1986), 57-106. MR 860811 Zbl 0646.46057

[19] M. Pimsner and S. Popa, Iterating the basic construction. Trans. Amer. Math. Soc. 310 (1988), 127-133. MR 965748 Zbl 0706.46047

[20] S. Popa, Classification of subfactors: the reduction to commuting squares. Invent. Math. 101 (1990), 19-43. MR 1055708 Zbl 0757.46054

[21] S. Popa, Classification of amenable subfactors of type II. Acta Math. 172 (1994), 163-255. MR $1278111 \mathrm{Zbl} 0853.46059$

[22] M. Takesaki, Theory of operator algebras I. Volume 124 of the Encyclopaedia of Mathematical Sciences. Springer-Verlag, Berlin, 2002. MR 1873025 Zbl 0436.46043

[23] Y. Watatani, Index for $C^{*}$-subalgebras. Amer. Math. Soc., Providence, RI, 1990. Google Books Bp2cmONVye0C MR 996807 Zbl 0697.46024

Received September 25, 2010

Vaughan F. R. Jones, Department of Mathematics, University of California, Berkeley, 970 Evans Hall \#3840, Berkeley, CA 94720-3840 U.S.A.

E-mail: vfr@math.berkeley.edu

David Penneys, Department of Mathematics, University of California, Berkeley,

970 Evans Hall \#3840, Berkeley, CA 94720-3840 U.S.A.

E-mail: dpenneys@math.berkeley.edu 